This manuscript is a non-peer reviewed EarthArXiv preprint that has been submitted for publication in Ocean Dynamics. If accepted, the final version of this manuscript will be available via the 'Peer-reviewed Publication DOI' link on the right-hand side of this webpage. 


\title{
Sedimentological data-driven bottom friction parameter estimation in modelling Bristol Channel tidal dynamics
}

\author{
Simon C. Warder · Athanasios Angeloudis . \\ Matthew D. Piggott
}

\begin{abstract}
Accurately representing the bottom friction effect is a significant challenge in numerical tidal models. Bottom friction effects are commonly defined via parameter estimation techniques. However, the bottom friction coefficient (BFC) can be related to the roughness of the sea bed. Therefore, sedimentological data can be beneficial in estimating BFCs. Taking the Bristol Channel and Severn Estuary as a case study, we perform a number of BFC parameter estimation experiments, utilising sedimentological data in a variety of ways. Model performance is explored through the results of each parameter estimation experiment, including applications to tidal range and tidal stream resource assessment. We find that theoretically derived sediment-based BFCs are in most cases detrimental to model performance. However, good performance is obtained by retaining the spatial information provided by the sedimentological data in the formulation of the parameter estimation experiment; the spatially varying BFC can be represented as a piecewise-constant field following the spatial distribution of the observed sediment types. By solving the resulting low-dimensional parameter estimation problem, we obtain good model performance as measured against tide gauge data. This approach appears well suited to modelling tidal range energy resource, which is of particular interest in the case study region. However, the applicability of this approach for tidal stream resource assessment is limited, since modelled tidal currents exhibit a strong localised response to the BFC; the use of piecewise-constant (and therefore discontinuous) BFCs is found to be detrimental to model performance for tidal currents.
\end{abstract}

Keywords Bottom friction - Manning coefficient - Calibration - Parameter estimation · Sedimentological data

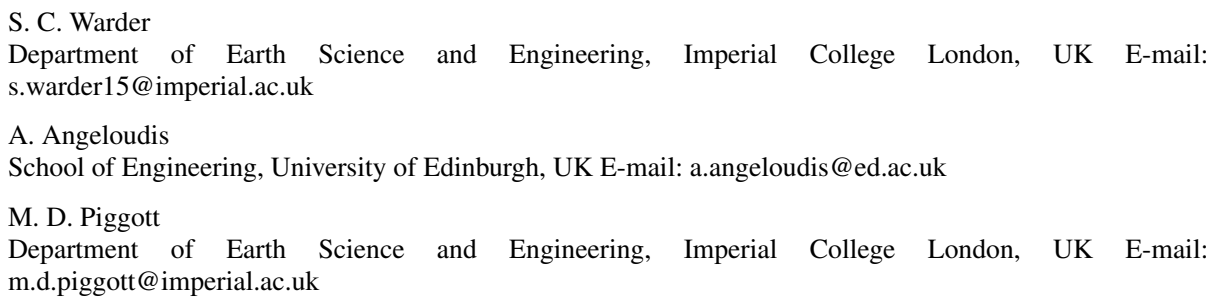




\section{Introduction}

Numerical modelling of tides in coastal and estuarine regions has applications in a wide variety of areas. An application of particular interest is marine renewable energy, with tidal modelling central to resource assessment for both tidal range [1,37,32] and tidal stream based energy projects $[50,53,55]$. With other applications of tidal models including sediment and pollutant transport [39,28], fisheries and ecosystems [34,57] and hazards such as storm surge $[12,23,54]$, accurate numerical modelling of tides is highly valuable.

However, such models are subject to a variety of uncertainty sources. Modelling errors arise from assumptions and simplifications in the governing equations, as well as discretisation errors, limitations in model resolution, and imperfect model inputs. One particular source of uncertainty, which is commonly addressed within the literature using parameter estimation methods, is the bottom friction coefficient (BFC). Friction between the ocean and the sea floor arises due to a boundary layer at the sea bed, and form drag due to bathymetry fluctuations. The process is not explicitly resolved in numerical models, and is instead treated as a parameterised process, via any of several formulations [60]. The value of the BFC therefore cannot be directly measured in the field, but can under certain assumptions be related to the roughness of the sea floor surface [46]. However, in addition to spatial variation due to bottom roughness, bottom friction parameters can also vary temporally (e.g. due to morphological changes [9] or seasonal changes in hydrological conditions [24]), as well as with mesh resolution, and a number of other physical or numerical variables [13]. For these reasons, bottom friction parameters are commonly treated via model calibration methods, where their value is determined by minimising the misfit between model outputs and observations, typically using data from tide gauges, acoustic Doppler current profilers (ADCPs) or satellite altimetry.

Approaches to model calibration within the literature vary widely in their complexity. Excluding studies dedicated to parameter estimation, the most common approach is to apply a spatially uniform BFC. In contrast, the highest-complexity approach is to allow the BFC to vary freely over the whole domain, and in this case it is common to supplement the observation data with a form of regularisation, to avoid the problem of over-fitting [35]. Intermediate complexity in the friction coefficient can be achieved via several approaches. [22] divide their model domain into regions of similar influence on the model-observation misfit using an adjoint gradient-based method, also taking into account the physical properties of the system. Another more common approach is the so-called independent points scheme, where the friction coefficient field is specified by interpolation between a selected set of 'independent points' [60,8]. The locations of these points can be distributed uniformly or according to physical features such as the bathymetry gradient [30]. Similarly, [48] divide their model domain by bathymetry contours in order to select a low-dimensional parameter space for their spatially varying BFC, while [36] propose the use of land use data to inform the BFC.

Alternatively, sedimentological data can be used for the purpose of constraining the spatial variation of the BFC, due to the underlying physical relationship between sediment type and the roughness of the sea bed, and hence the value of the friction coefficient. [33] directly apply Manning coefficients derived from sedimentological data within a model of the Irish Sea, supplemented by a localised BFC enhancement around a region of interest which they tune for optimal model performance. Similarly, [17] utilise sedimentological data to derive a spatially varying quadratic drag parameter for a tidal stream power application off the coast of Brittany, and subsequently perform a sensitivity analysis with respect to the roughness length assigned to one of the sediment types. 
Within this study, we explore the use of sedimentological data within a BFC parameter estimation problem. We perform a number of parameter estimation experiments, utilising such data in different ways. By comparing model performance using the results of each parameter estimation experiment, the objective is to arrive at recommendations regarding the use of sedimentological data in informing bottom friction parameters.

A description of the case study region, numerical model and data sources can be found in section 2. Section 3 presents the Bayesian inference parameter estimation method used, which is based on M2 and S2 harmonic amplitude and phase data at 15 tide gauges within the model domain. Calibration and validation results can be found in sections 4 and 5, respectively. In section 6, we apply the calibrated model to the estimation of tidal range energy resource. The case study is primarily motivated by tidal range energy, and hence the main focus is on model comparisons with tide gauge data. However, in section 7 we explore model performance using tidal current observations from an ADCP, as a step towards application of the calibrated model to tidal stream resource assessment. Finally, a discussion and conclusions can be found in sections 8 and 9 , respectively.

\section{Description of model and data}

\subsection{Model study region}

The model study region consists of the Bristol Channel and Severn Estuary, situated to the south-west of the UK, as shown in Fig. 1. A macrotidal inlet offering significant tidal range energy resource [2], the Bristol Channel is also of interest for tidal stream energy [50]. Accurate tidal models of the region are also relevant to flood risk studies (e.g. [31]) due to its susceptibility to storm surge $[40,58]$. A number of flooding events have occurred in the area in recent years, for example in the Somerset Levels [45], and future flood risk is linked to climate change [41]. The region is also to be used as a case study for a calibration and validation phase of the forthcoming SWOT mission [38].

The tidal dynamics in the region are dominated by the M2 and S2 constituents, whose average amplitudes within the Bristol Channel are around $3.5 \mathrm{~m}$ and $1.2 \mathrm{~m}$, respectively. Within this work we also utilise observations of the N2 and M4 constituents, whose amplitudes are around 0.6 and $0.2 \mathrm{~m}$, respectively.

\subsection{The Thetis numerical model}

Within this work we use Thetis, an unstructured-mesh finite element coastal ocean model [26] which utilises the Firedrake finite element code generation framework [42]. We employ Thetis in its two-dimensional configuration (as in [51]), which solves the nonlinear shallow water equations given by

$$
\frac{\partial \eta}{\partial t}+\nabla \cdot(H \mathbf{u})=0
$$

$$
\frac{\partial \mathbf{u}}{\partial t}+\mathbf{u} \cdot \nabla \mathbf{u}+\mathbf{F}_{\mathbf{C}}+g \nabla \eta=-\frac{\tau_{b}}{\rho H}+\nabla \cdot\left(v\left(\nabla \mathbf{u}+\nabla \mathbf{u}^{T}\right)\right),
$$

where $\eta$ is the free surface elevation, $H=\eta+h$ is the total water depth, $h$ is the bathymetry, $\mathbf{u}$ is the two-dimensional depth-averaged velocity, $\mathbf{F}_{\mathbf{C}}$ is the Coriolis force, $g$ is the acceleration due to gravity, $\rho$ is the water density (which is taken as a constant), $\tau_{b}$ is the bottom 
stress due to friction between the ocean and sea bed, and $v$ is the eddy viscosity (which we assign a constant value of $1 \mathrm{~m}^{2} \mathrm{~s}^{-1}$ ). We parameterise the bottom friction $\tau_{b}$ via a Manning's $n$ formulation

$$
\frac{\tau_{b}}{\rho}=\frac{g n^{2}}{H^{\frac{1}{3}}}|\mathbf{u}| \mathbf{u}
$$

where $n$ is the Manning coefficient (units $\mathrm{s} \mathrm{m}^{-1 / 3}$ ). For the purposes of model calibration within this work, $n$ depends on the sediment type found on the ocean bed (see section 2.3).

Since the Bristol Channel and Severn Estuary contain significant intertidal regions, we include wetting and drying within Thetis using the scheme of [25], which we summarise here. Under this scheme, a modification is applied dynamically to the bathymetry in order to avoid negative water depth. The modified bathymetry is given by

$$
\tilde{h}=h+f(H),
$$

such that the modified water depth is similarly given by

$$
\tilde{H}=H+f(H) .
$$

The implementation of this scheme simply requires this modified depth $\tilde{H}$ to be substituted for $H$ in the governing equations (1). The function $f(H)$ is chosen such that the modified water depth $\tilde{H}$ is always positive. Following [25], we use

$$
f(H)=\frac{1}{2}\left(\sqrt{H^{2}+\alpha^{2}}-H\right),
$$

where $\alpha$ is a wetting-drying parameter which controls the transition from wet to dry regions, and is user defined. In general, smaller values of $\alpha$ result in more accurate results, but there exists a minimum stable value which is related to the mesh element size. In all Thetis simulations presented herein, $\alpha$ is taken to be $1 \mathrm{~m}$; this value was found through preliminary experiments (not shown) to be close to the minimum stable value for the selected mesh.

Mesh generation was performed using the Python package qmesh [5], which interfaces the mesh generator Gmsh [14]. The mesh, shown in Fig. 1, adopts a UTM30 coordinate projection, and uses a variable mesh element size from $250 \mathrm{~m}$ in the inner Bristol Channel, to $8 \mathrm{~km}$ in open regions, resulting in a total of 42,862 triangular elements. Coastline data for mesh generation is from the Global Self-consistent, Hierarchical, High-resolution Geography Database (GSHHG) [56]. Thetis is run using a $\mathrm{P}_{1}^{\mathrm{DG}}-\mathrm{P}_{1}^{\mathrm{DG}}$ discretisation, with a Crank-Nicolson timestepping scheme with a timestep $\Delta t=100 \mathrm{~s}$. The bathymetry is from 6 arcsecond resolution data available from Digimap [10], and is shown in Fig. 2.

Tidal dynamics are introduced through a Dirichlet boundary condition for the surface elevation $\eta$ at the ocean boundary, extracted from the TPXO database [11]. The location of the ocean boundary of the model domain was selected to be in reasonably deep water, to minimise the influence of errors in this tidal boundary forcing data. The tidal dynamics within the Bristol Channel are dominated by the M2 and S2 constituents (with amplitudes in excess of $1 \mathrm{~m}$ ), with some contribution from the N2, K2 and M4 constituents (amplitudes in the $10 \mathrm{~s}$ of $\mathrm{cm}$ ), and no other constituents above $10 \mathrm{~cm}$ amplitude. Due to their similar frequencies and the constraints of the Rayleigh criterion, the K2 and S2 constituents require long periods of observation/simulation to be resolved, and we therefore neglect the K2 constituent. The M2, S2, N2 and M4 constituents are therefore the focus of model-observation comparisons we perform within this study, and thus we use the same four constituents to force the model at its boundaries. The shallow-water M4 constituent is mostly generated within the model domain and has small amplitude on the boundaries, but is nevertheless included in the boundary forcing. Model runs span a 5-day spinup period, followed by two full spring-neap cycles (approximately one month). 


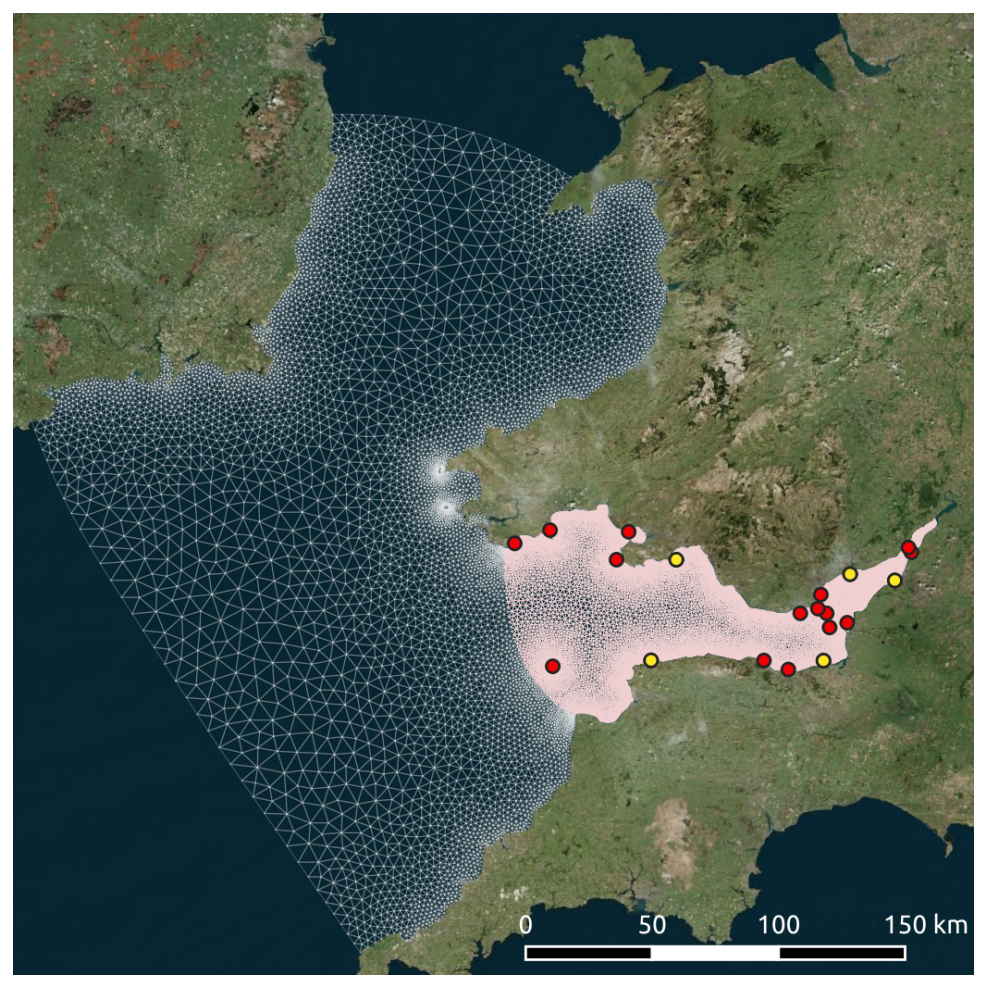

Fig. 1: Mesh used for all simulations within this paper. Red circles: locations where harmonic analysis data are available. M2 and S2 harmonic data at these locations are used within this work for model calibration, and N2 and M4 data for validation. Yellow circles: BODC tide gauge locations, where timeseries data is available. M2 and S2 data derived from these timeseries are used within this work for validation. The coloured region of the mesh indicates where a spatially variable friction coefficient is applied.

\subsection{Parameterising the Manning coefficient}

We employ a parameter estimation method in order to calibrate the model with respect to the spatially varying Manning coefficient, $n$. In order to constrain the parameter's spatial variation, we use sediment maps within the model domain. In an approach similar to [33], we use data from the British Geological Survey [7], which indicates the type of sediment found at each point in the domain. The distribution of sediment types is shown in Fig. 3, and summarised in Table 1.

The Manning coefficient can in principle be determined directly from the sediment type found at a given location, via a lookup table for the median sediment grain size for the corresponding sediment type. Denoting the median grain size $d_{50}$ (in $\mathrm{m}$ ), the corresponding theoretical Manning coefficient is given by

$$
n\left(d_{50}\right)=0.04 \sqrt[6]{2.5 d_{50}}
$$

[46]. This results in the set of Manning coefficients detailed in Table 1, which are consistent with standard sediment-based values from other sources (e.g. [4]). Throughout this paper, we 


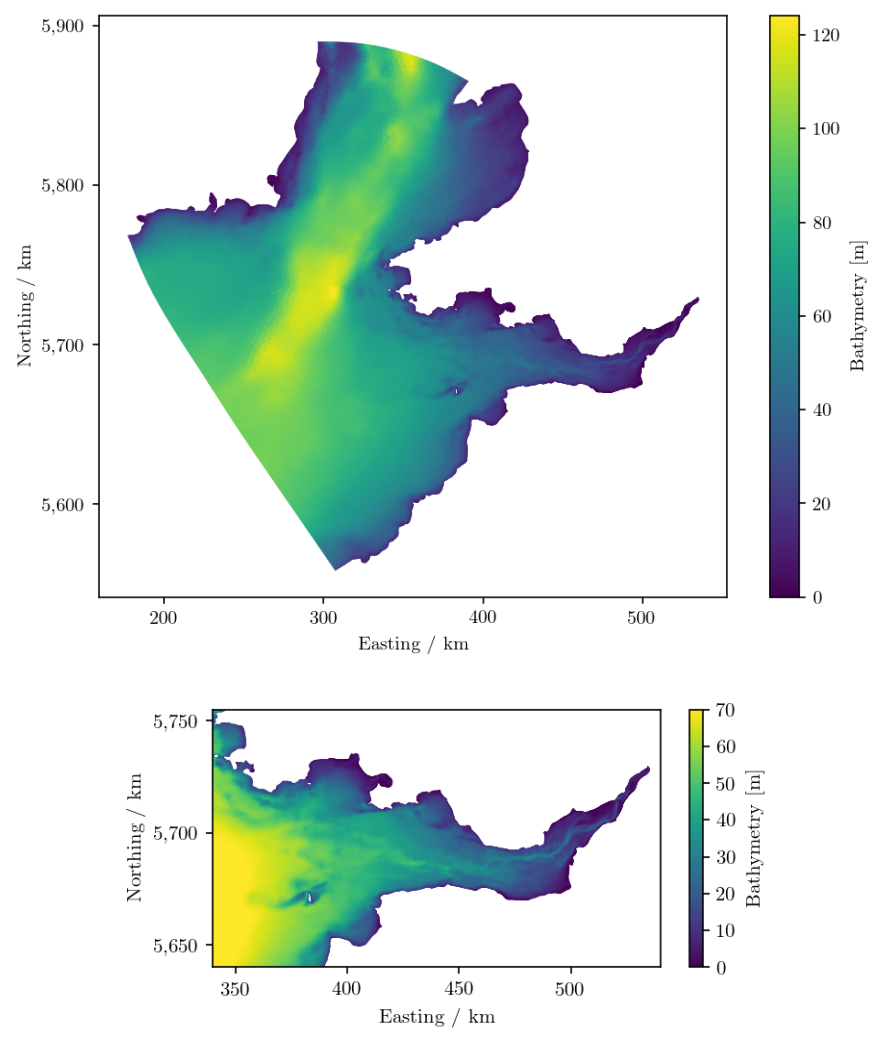

Fig. 2: Top: Bathymetry over the full model domain. Bottom: Bathymetry within the Bristol Channel and Severn Estuary. Coordinates are in the UTM30 projection.

refer to the set of Manning coefficients computed via Eq. (6) as the 'standard' or 'theoretical' sediment-based parameters.

However, there is uncertainty inherent in the direct application of Manning coefficients computed as above. The bed friction term in the model governing equations must ideally account for unresolved bathymetry and bedforms, which are not accounted for within equation (6). Additionally, due to numerical dissipation, it may be the case that the optimal friction coefficients within a numerical model are smaller than those corresponding to the true properties of the sea bed [13]. Therefore, even when sediment data is available (as is the case here), it is common within the numerical modelling literature to perform model calibration with respect to the bottom friction coefficient. Nevertheless, the availability of sediment data can be used to constrain the spatial variation of the bottom friction parameter, in order to reduce the dimension of the parameter space for parameter estimation.

Within this work, we perform several parameter estimation experiments labelled A, B, $\mathrm{C} 1$ and $\mathrm{C} 2$ and described below. In each case, the Manning coefficient in the outer region of the model domain, indicated by the white region of the mesh in Fig. 1, is held constant at $n=0.025 \mathrm{~s} \mathrm{~m}^{-1 / 3}$. Since model-observation comparisons are made only within the Bristol Channel, the value for $n$ within this outer region was found to have only a very weak influence on the model performance metrics, and a value of $n=0.025 \mathrm{~s} \mathrm{~m}^{-1 / 3}$ was found through 
preliminary experiments (not shown) to produce adequate results. The value for $n$ inside the Bristol Channel (coloured region in Fig. 1) is described below for each experiment:

\section{Experiment A: Estimation of a spatially uniform Manning coefficient.}

The simplest approach is to discard the sediment data entirely, and estimate only a spatially uniform Manning coefficient (i.e. a single value), $n_{0}$. This is a commonly taken approach within the literature, especially where more advanced model calibration is not directly the focus of the work.

Experiment B: Estimation of a scaling factor for the standard sediment-based Manning coefficients.

An alternative is to scale the Manning coefficients given by Eq. (6) by a spatially uniform factor $\gamma$, such that

$$
n\left(d_{50}\right)=0.04 \gamma \sqrt[6]{2.5 d_{50}}
$$

The parameter estimation problem is to determine the optimal value for $\gamma$. The motivation for this approach is that the sediment-based Manning coefficient is likely to overestimate the required bottom friction, due to the presence of numerical dissipation, but that the relative values of the Manning coefficients based on the sediment data may still be appropriate. This approach results in the same number of degrees of freedom (one) in the parameter estimation problem as experiment A, but incorporates a priori knowledge about the physical process of bottom friction.

\section{Experiment C: Direct estimation of a small number of Manning coefficients correspond-} ing to groups of sediment classes.

The third approach we take within this work is to estimate three Manning coefficients $\left(n_{1}, n_{2}, n_{3}\right)$, each corresponding to a group of sediment types. We choose to group the sediment types into approximately equal area (see Table 1), such that $n_{1}$ corresponds to sediment types $1-4, n_{2}$ to types $5-8$, and $n_{3}$ to types $9-13$. This grouping is shown in Fig. 4. While we could have used the sediment data to divide the domain into more than three subdomains, this would result in large variation in subdomain area, with parameters corresponding to small domain areas unlikely to be well constrained by the observations. We further subdivide this experiment into two. In experiment C1, we use uniform priors for each parameter within the Bayesian inference parameter estimation algorithm we employ. In experiment C2, we use the standard sediment-derived Manning coefficients to construct Gaussian prior distributions for each parameter.

Alongside the results of each of the above parameter estimation experiments, we also present results based on a uniform Manning coefficient of $0.025 \mathrm{~s} \mathrm{~m}^{-1 / 3}$ throughout the model domain. This value is somewhat arbitrary, but falls within the commonly used range of uniform Manning coefficients within the literature. Results using this uniform BFC represent a useful benchmark against which to compare the performance resulting from each of the above parameter estimation experiments.

\subsection{Observation data}

We use data from two sources for the purposes of model calibration and validation, as indicated in Fig. 1:

(i) 15 locations at which tidal harmonic data is available (National Oceanography Centre, personal communication 2018), which are shown as red circles in Fig. 1. We use 


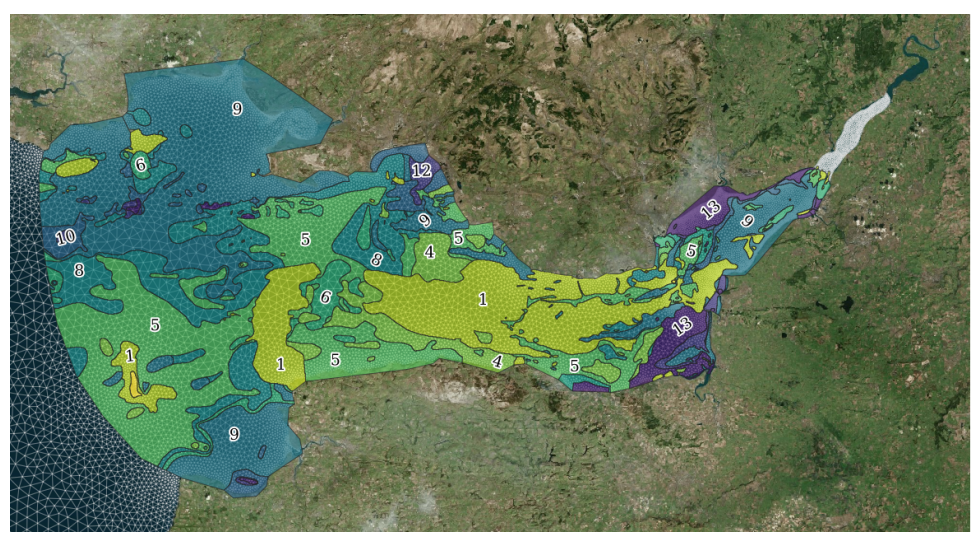

Fig. 3: Spatial distribution of sediment types within the Bristol Channel, from the British Geological Survey [7]. See also Table 1.

Table 1: Sediment types defined by the British Geological Survey [7], sorted by roughness length. Theoretical values for the Manning coefficient $n$ are calculated from Eq. (6). See Fig. 3 for the spatial distribution of the sediment types. Based on [33].

\begin{tabular}{c|c|c|c} 
Sediment ID & Sediment name & Area of Bristol Channel $\left[\mathrm{km}^{2}\right]$ & Theoretical $n\left[\mathrm{sm}^{-1 / 3}\right]$ \\
\hline 1 & Bedrock & 1090 & 0.049 \\
2 & Boulder & 0 & 0.041 \\
3 & Cobble & 0 & 0.033 \\
4 & Very coarse gravel & 334 & 0.0275 \\
5 & Coarse gravel & 1465 & 0.0245 \\
6 & Medium gravel & 227 & 0.022 \\
7 & Fine gravel & 34 & 0.020 \\
8 & Very coarse sand & 831 & 0.018 \\
9 & Coarse sand & 1775 & 0.016 \\
10 & Medium sand & 192 & 0.014 \\
11 & Fine sand & 1 & 0.0125 \\
12 & Very fine sand & 87 & 0.011 \\
13 & Silt, clay, mud & 190 & 0.0095
\end{tabular}

the M2 and S2 harmonic amplitudes and phases at these locations for the model calibration. N2 and M4 data at these locations are used for model validation. The tidal harmonics at each observation location were computed from tide gauge records between one month and one year in length, between 1960 and 1980.

(ii) Five tide gauges where quality controlled timeseries surface elevation data are available from the British Oceanographic Data Centre (BODC). These locations are shown in Fig. 1 by yellow circles. The tidal constituent data we use at these locations is from a harmonic analysis of observations spanning a 10 year period from 1997 . We use M2 and S2 amplitude and phase observations at these locations for further validation of the calibrated models. 


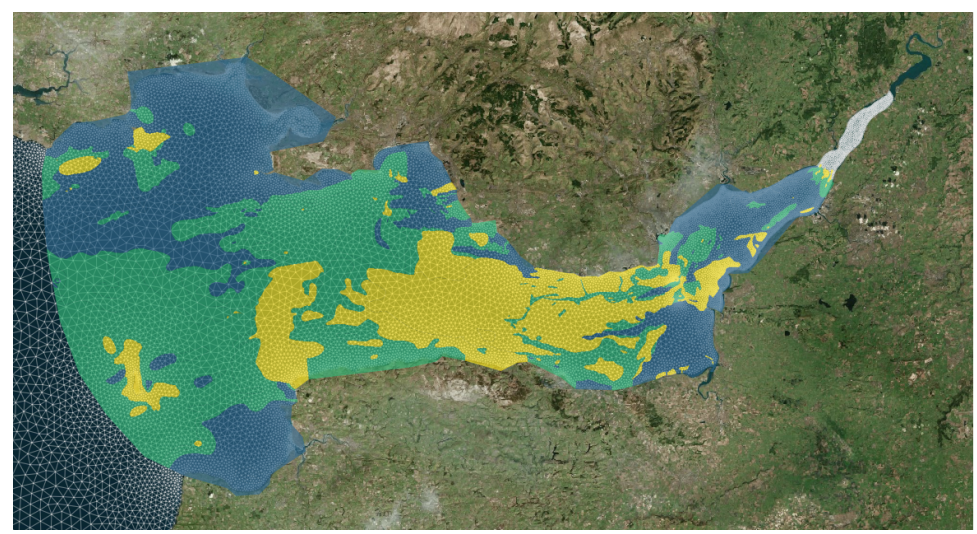

Fig. 4: Grouping of sediment classes for the purposes of parameter estimation experiments $\mathrm{C} 1$ and $\mathrm{C} 2$. Yellow corresponds to parameter $n_{1}$, green $n_{2}$ and blue $n_{3}$. In any regions where sediment data is unavailable, the default Manning coefficient of $n=0.025 \mathrm{~s} \mathrm{~m}^{-1 / 3}$ is applied.

\section{Parameter estimation method}

There exist a large number of algorithms within the literature for estimating unknown bottom friction parameters. In the simple one-dimensional case (i.e. using a spatially uniform BFC), it is common to employ a simple grid search. This involves simply running the numerical model with a small number of different BFC values, and selecting the value which minimises a given measure of model-observation misfit. However, this approach scales poorly with the number of parameters to be estimated. The high-complexity approach (estimating an independent BFC value at every mesh node) typically requires numerical adjoint models, which constitute an efficient technique for evaluating gradients of model outputs (typically a functional representing the model-observation misfit) with respect to the control parameters, thus facilitating the use of gradient-based optimisation methods for performing model calibration [35]. The use of intermediate-complexity BFC parameterisations is compatible with a number of approaches, with adjoint [60] or other gradient-based methods [47], Kalman filters [36,43] and Markov Chain Monte Carlo (MCMC) methods [18,48] all employed within the literature.

Within this work, we take a Bayesian inference approach via an MCMC algorithm. We utilise a Gaussian process emulator as an efficient surrogate for the full numerical model. This is necessary because the MCMC algorithm requires large numbers of model runs (typically $\mathscr{O}\left(10^{6}\right)$ ), which is not feasible with the full numerical model. While our numerical model does have an adjoint model available, the size of the parameter estimation problems we solve within this work are relatively small and do not warrant adjoint methods. Kalman filter approaches typically require some tuning of algorithm parameters for optimal performance [43]. The MCMC approach however is fairly straightforward and well suited to the size of the problem considered here. Its results are simple to interpret, and also yield a direct estimate of the uncertainty in the estimated parameters.

The following exposition of the Bayesian inference algorithm proceeds for parameter estimation experiment $\mathrm{C}$, since this is the most general case (estimating the greatest number 
of parameters). The application of the method to experiments A and B requires only minor adaptation.

\subsection{Bayesian inference}

Within this work, the observation data we use for calibration consists of M2 and S2 harmonic amplitudes and phases at 15 tide gauge locations (as indicated by the red circles in Fig. 1).

We denote these four observations types by $j=1,2,3,4$, corresponding to M2 amplitude, S2 amplitude, M2 phase and S2 phase, respectively. The observation data is thus represented by four vectors $\mathbf{y}_{j}$, each of length $N=15$. For compactness, we denote the full set of observations $Y$, a matrix with shape $(4 \times N)$, whose rows are given by the vectors $\mathbf{y}_{j}$. The corresponding model outputs for observation type $j$ are denoted $\mathbf{f}_{j}(\mathbf{n})$. Bayes' theorem gives

$$
\Pi(\mathbf{n} \mid Y) \propto L(Y \mid \mathbf{n}) \prod_{i=1}^{3} q_{i}\left(n_{i}\right),
$$

where $\Pi$ is the posterior distribution of the parameters $\mathbf{n}=\left(n_{1}, n_{2}, n_{3}\right)$ given the observed data $Y, L$ is the likelihood of observing the outputs $Y$ given the parameters $\mathbf{n}$, and $q_{i}$ is the prior distribution for each of the parameters $n_{i}$.

The likelihood $L$ is estimated from the numerical model. For observation type $j$, we assume that the model-observation discrepancies, which are the components of the vector $\mathbf{y}_{j}-\mathbf{f}_{j}(\mathbf{n})$, are independent and identically distributed variables with zero mean and variance $\sigma_{j}^{2}$. The likelihood $L(Y \mid \mathbf{n})$ is then given by

$$
L(Y \mid \mathbf{n})=\prod_{j=1}^{4}\left[\left(2 \pi \sigma_{j}^{2}\right)^{-N / 2} \exp \left(-\frac{1}{2} \frac{\left|\mathbf{y}_{j}-\mathbf{f}_{j}(\mathbf{n})\right|^{2}}{\sigma_{j}^{2}}\right)\right] .
$$

Since the $\sigma_{j}^{2}$ values are unknown a priori, they are treated as hyperparameters, i.e. they are included as additional parameters to be inferred by the inversion algorithm. We denote the full vector of unknowns $\theta=\left(n_{1}, n_{2}, n_{3}, \log \sigma_{1}^{2}, \log \sigma_{2}^{2}, \log \sigma_{3}^{2}, \log \sigma_{4}^{2}\right)$, and the full posterior distribution is therefore given by

$$
\Pi(\boldsymbol{\theta} \mid Y) \propto \prod_{j=1}^{4}\left[\left(2 \pi \sigma_{j}^{2}\right)^{-N / 2} \exp \left(-\frac{1}{2} \frac{\left|\mathbf{y}_{j}-\mathbf{f}_{j}(\mathbf{n})\right|^{2}}{\sigma_{j}^{2}}\right)\right] \prod_{i=1}^{3} q_{i}\left(n_{i}\right) \prod_{j=1}^{4} q_{j}\left(\log \sigma_{j}^{2}\right),
$$

where $q_{j}\left(\log \sigma_{j}^{2}\right)$ is the prior distribution of $\log \sigma_{j}^{2}$.

\subsubsection{Priors}

For parameter estimation experiments $\mathrm{A}, \mathrm{B}$ and $\mathrm{C} 1$, we use uniform priors for the corresponding control parameters. This is equivalent to setting $q_{i}\left(n_{i}\right)=1$ in Eq. (10) (the normalisation is not important). For parameter estimation experiment $\mathrm{C} 2$, we use the 'standard' sediment-based Manning coefficients of Table 1 to construct Gaussian priors for each of the Manning coefficients. That is, the priors are given by

$$
q_{i}\left(n_{i}\right)=\frac{1}{s_{i} \sqrt{2 \pi}} \exp \left(-\frac{1}{2} \frac{\left(n_{i}-\mu_{i}\right)^{2}}{s_{i}^{2}}\right),
$$


Table 2: Mean $(\mu)$ and standard deviation $(s)$ for the Manning coefficient priors in experiment $\mathrm{C} 2$.

\begin{tabular}{c|c|c} 
Manning coefficient & $\mu_{i} / \mathrm{sm}^{-1 / 3}$ & $s_{i} / \mathrm{sm}^{-1 / 3}$ \\
\hline$n_{1}$ & 0.0395 & 0.0135 \\
$n_{2}$ & 0.0215 & 0.0045 \\
$n_{3}$ & 0.013 & 0.004
\end{tabular}

where $\mu_{i}$ and $s_{i}$ are the mean and standard deviation of the prior distributions, whose values are summarised in Table 2.

For the unknown variances $\sigma_{j}^{2}$, the only prior constraint is that they must be positive. For all parameter estimation experiments within this study, we follow the approach of [48] and assume Jeffreys priors [44], such that

$$
q_{j}\left(\log \sigma_{j}^{2}\right)=\frac{1}{\sigma_{j}^{2}} .
$$

\subsection{Markov Chain Monte Carlo algorithm}

A technique for sampling the posterior distribution given by Eq. (10) is the Markov Chain Monte Carlo (MCMC) method, which has the advantage that the constant of proportionality in the equation need not be determined. We use an implementation of the Random Walk Metropolis Hastings MCMC algorithm [21], which is given by Algorithm 1. The algorithm requires the selection of an appropriate proposal distribution covariance matrix, $\Sigma_{\text {step }}$, governing the size of the random steps within the parameter space. We set

$$
\Sigma_{\text {step }}=\operatorname{diag}\left(0.001^{2}, 0.001^{2}, 0.001^{2}, 0.1^{2}, 0.1^{2}, 0.1^{2}, 0.1^{2}\right)
$$

so that the random steps in each of the Manning coefficients have zero mean and a standard deviation of $0.001 \mathrm{~s} \mathrm{~m}^{-1 / 3}$, and the random steps in each value of $\log \sigma_{j}^{2}$ have zero mean and a standard deviation of 0.1 . These step sizes were found to give satisfactory results, without the need for an adaptive MCMC algorithm.

In the results presented here, we take $M=10^{6}$ samples, discarding the first $2 \times 10^{5}$ as a burn-in period, and the resulting chain of values $n^{[k]}$ generated by the MCMC algorithm constitute samples from the posterior distribution. The mean of these samples is taken as the best estimate of the parameter values.

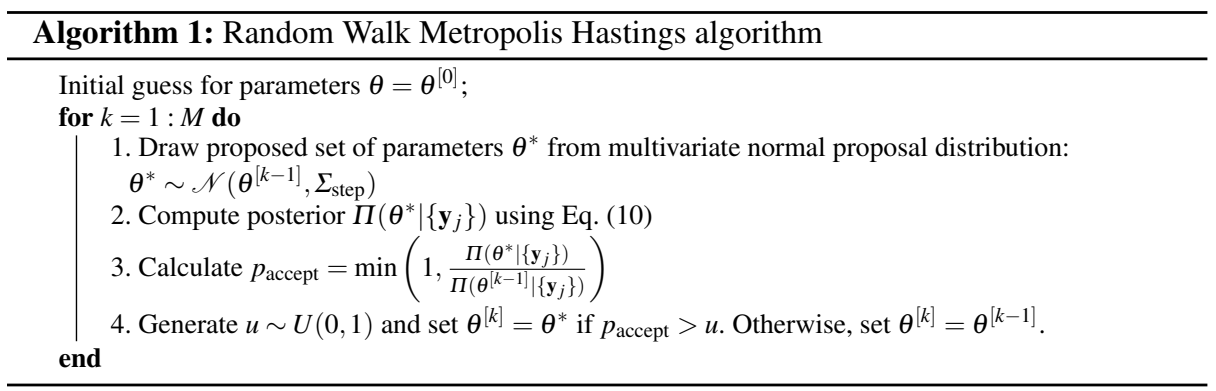




\subsection{Gaussian process emulation}

We employ a Gaussian process emulator (GPE) as a computationally inexpensive surrogate for the full numerical model. For parameter estimation experiment C, this GPE is trained using 40 model runs with Manning coefficient samples drawn from uniform prior distributions in the range [0.01, 0.05], using Latin Hypercube Sampling to evenly sample the three-dimensional parameter space. Experiment $\mathrm{A}$ is a simplified version of experiment $\mathrm{C}$, and can therefore utilise the same GPE. For experiment B, where the objective is to estimate the scaling parameter $\gamma$ (see Eq. (7)), the GPE is trained using 10 samples for $\gamma$ drawn uniformly between 0.55 and 1.0, inclusive. Values for $\gamma$ smaller than 0.55 resulted in model instabilities due to the very low friction coefficients in some regions. Once trained, the GPE is substituted for $\mathbf{f}(\mathbf{n})$ within the MCMC algorithm described above. Within this study, we use the Python package GPy [15] for the construction of GPEs.

The use of a GPE in place of the full Thetis model introduces additional uncertainty. However, this uncertainty can be directly estimated by the GPE. The GPE-introduced covariances were typically around $10^{-6} \mathrm{~m}^{2}$ for emulated amplitudes, and $2 \times 10^{-3^{\circ 2}}$ for emulated phases. Since the model-observation variances $\left(\sigma_{j}^{2}\right.$ in the above description of the Bayesian inference) were typically around $25 \mathrm{~cm}^{2}$ for amplitudes, and $6.25^{\circ 2}$ for phases, the additional uncertainty introduced by the GPEs is small, and can be neglected.

\section{Calibration results}

\subsection{Optimal parameters}

The optimal Manning coefficient fields for each parameter estimation experiment are shown in Fig. 7. Note that in all cases, the value of the Manning coefficient outside the Bristol Channel takes a fixed value of $n=0.025 \mathrm{sm}^{-1 / 3}$, as described in section 2.3. We make further comments on the results from each experiment below.

Experiment A: uniform parameter inside Bristol Channel

The optimal uniform parameter within the Channel (and its uncertainty) is given by $n_{0}=$ $0.0274 \pm 0.0003$. This value lies within the range of commonly used uniform parameter values in the literature.

Experiment B: scaling of 'standard' sediment-based parameters

The MCMC algorithm returns a scaling parameter $\gamma=0.813 \pm 0.013$. This is consistent with the expectation that the 'standard' sediment-based parameters are too strongly dissipative, due to the presence of numerical dissipation.

\section{Experiment $\mathrm{C} 1$ : three-dimensional parameter space, uniform priors}

The values for each Manning coefficient returned by the MCMC algorithm are $n_{1}=$ $0.032 \pm 0.002, n_{2}=0.021 \pm 0.007, n_{3}=0.025 \pm 0.003$. The marginal posterior distributions for each parameter are shown in Fig. 5. Each marginal distribution is obtained by integrating the full posterior distribution over two of the parameters, leaving the marginal PDF for each parameter individually. The relative magnitudes of the Manning coefficients returned by this experiment are unexpected; given the sediment types corresponding to each parameter, we would expect $n_{1}>n_{2}>n_{3}$. We note that the posterior distribution for $n_{2}$ is very broad. The parameter estimation results are therefore not necessarily inconsistent with this expectation, but the means of the distributions do not fall in the expected order. 

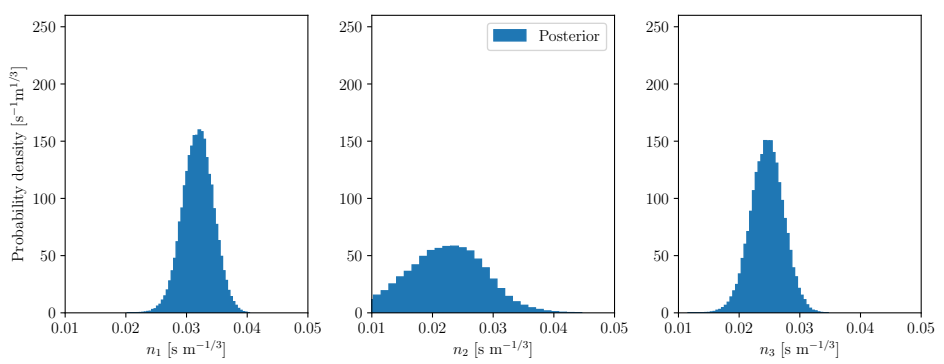

Fig. 5: Marginal posterior distributions for each parameter $n_{i}$, from experiment $\mathrm{C} 1$.
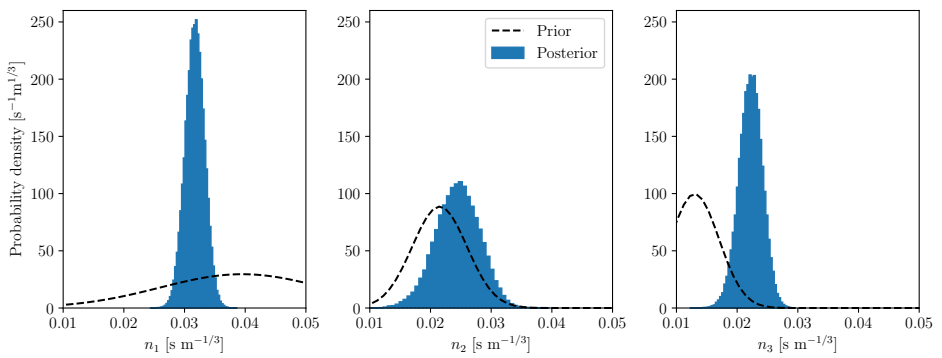

Fig. 6: Marginal posterior distributions for each parameter $n_{i}$, from experiment C2. Dotted lines indicate the prior distributions for each parameter.

\section{Experiment C2: three-dimensional parameter space, Gaussian priors}

The values for each Manning coefficient returned by the MCMC algorithm are $n_{1}=$ $0.0317 \pm 0.0016, n_{2}=0.024 \pm 0.004, n_{3}=0.0222 \pm 0.0019$. The marginal posterior distributions for each parameter are shown in Fig. 6, along with the prior distributions. The prior distribution for $n_{1}$ is very broad, with the observation data able to achieve a far tighter constraint. For all three parameters, the posterior distributions are narrower than for experiment $\mathrm{C} 1$, due to the additional constraints provided by the priors. Note also that the influence of the priors is sufficient for the parameters to fall in the expected order $\left(n_{1}>n_{2}>n_{3}\right)$, in contrast to experiment $\mathrm{C} 1$.

\subsection{Performance against calibration dataset}

In this section, we summarise the performance of the model with the Manning coefficient field resulting from each parameter estimation experiment, as measured against the calibration dataset (locations indicated by red circles in Fig. 1). Results presented here are based on runs of the full numerical model (not the GPE). The M2 and S2 amplitude and phase RMSEs achieved with each coefficient field are summarised in Table 3.

As described in section 2.3, the uniform BFC of $0.025 \mathrm{~s} \mathrm{~m}^{-1 / 3}$ is used as a benchmark, with which we can compare model performance using the other BFC fields. The 'standard' sediment-based parameters perform very poorly, with significantly greater RMSEs than the 

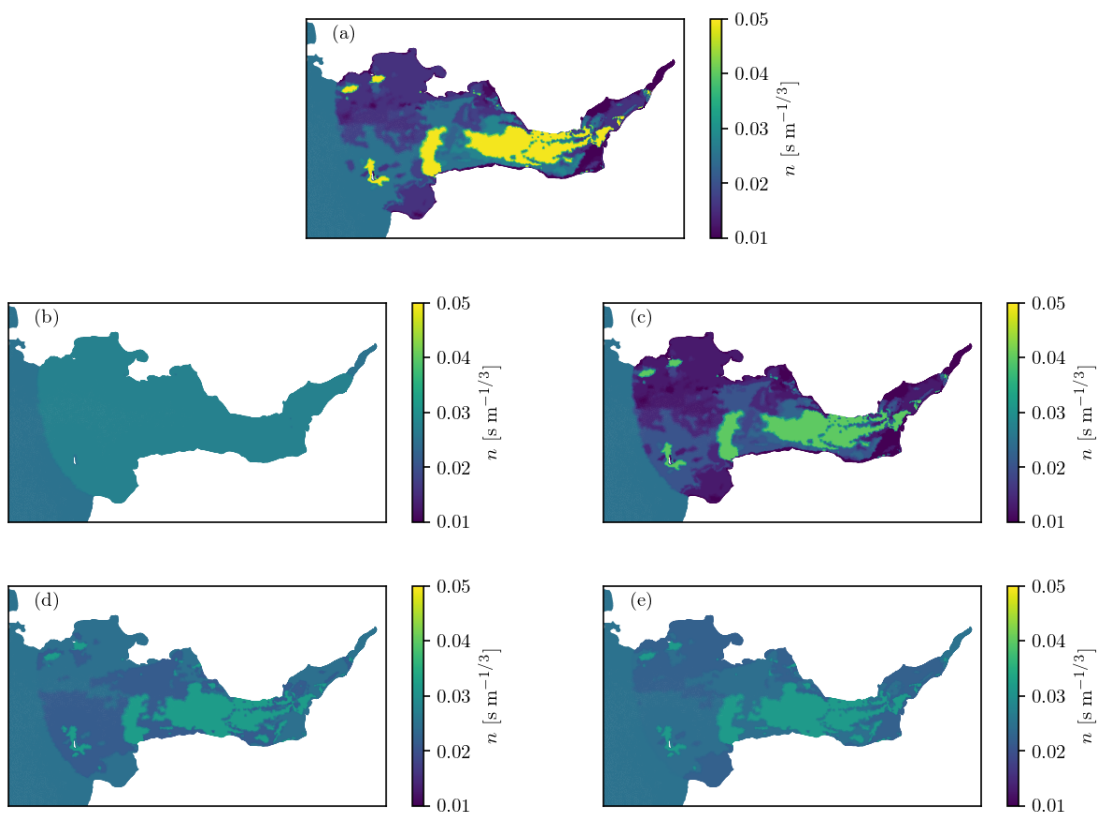

Fig. 7: Manning coefficient fields used for model validation. (a) Standard sediment-based parameters. (b) Result of experiment A. (c) Result of experiment B. (d) Result of experiment C1. (e) Result of experiment C2.

benchmark run. Experiment A (optimal uniform BFC) performs well, and achieves the overall lowest amplitude and phase RMSEs for the S2 constituent, while the greatest improvement over the benchmark run is for the M2 amplitude. Experiment B does not perform as well as experiment $\mathrm{A}$, suggesting that the direct use of sediment-derived coefficients (even when scaled) is detrimental to model performance. Experiments $\mathrm{C} 1$ and $\mathrm{C} 2$ both perform well. Experiment $\mathrm{C} 1$ performs best overall, since its RMSEs are all within $0.1 \mathrm{~cm}$ or $0.1^{\circ}$ of the lowest achieved in all cases. This is to be expected, since experiment $\mathrm{C} 1$ uses the greatest number of degrees of freedom in representing the Manning coefficient, with the fewest additional constraints (whereas experiment $\mathrm{C} 2$ includes Gaussian priors for the unknown parameters).

Fig. 8 compares the modelled and observed M2 and S2 amplitudes and phases for both the 'standard' and experiment $\mathrm{C} 1$ cases. These results demonstrate the excessive dissipation due to the 'standard' friction coefficients, resulting in underestimated amplitudes. Figure 9 indicates the spatial distribution of the M2 amplitude errors within the Bristol Channel using the 'standard' parameters, and shows the increasing magnitude of the model errors further into the channel, where the amplitude increases due to resonance. The result of experiment $\mathrm{C} 1$ exhibits significantly reduced scatter, corresponding to the reduced RMSEs summarised in Table 3. 
Table 3: Root mean squared errors (RMSEs) of the modelled M2 and S2 amplitudes $(\alpha)$ and phases $(\phi)$, for each Manning coefficient field, aggregated across the calibration tide gauges (red circles in Fig. 1). Figures in bold indicate the best performance.

\begin{tabular}{c|c|c|c|c}
\multirow{2}{*}{ Manning coefficient field } & \multicolumn{4}{|c}{ RMSE } \\
\cline { 2 - 5 } & $\mathrm{M} 2 \alpha[\mathrm{cm}]$ & $\mathrm{M} 2 \phi\left[^{\circ}\right]$ & $\mathrm{S} 2 \alpha[\mathrm{cm}]$ & $\mathrm{S} 2 \phi\left[^{\circ}\right]$ \\
\hline 'Standard' sediment-based parameters & 22.6 & 8.6 & 15.2 & 9.2 \\
Experiment A & 4.9 & 2.6 & $\mathbf{6 . 1}$ & $\mathbf{3 . 0}$ \\
Experiment B & 9.9 & 3.8 & 7.3 & 5.0 \\
Experiment C1 & 3.4 & $\mathbf{2 . 5}$ & $\mathbf{6 . 1}$ & 3.1 \\
Experiment C2 & $\mathbf{3 . 3}$ & 2.7 & 6.3 & 3.3 \\
\hline Uniform $n=0.025 \mathrm{sm}^{-1 / 3}$ & 11.4 & 2.9 & 6.3 & 5.1
\end{tabular}
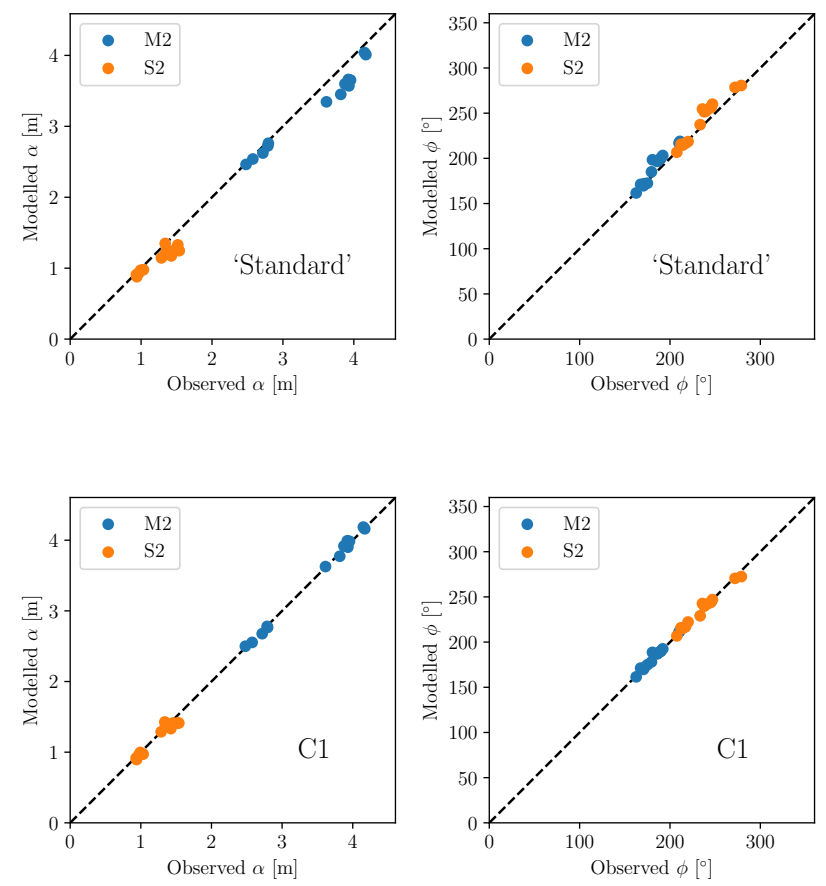

Fig. 8: Scatter plots of modelled M2 and S2 amplitude and phase, against observed values. Top: using 'standard' sediment-based parameters. Bottom: using result from experiment C1. The 'standard' parameters systematically underestimate the observed amplitudes.

\section{Validation of calibrated models}

Section 4.2 summarised model performance against the set of data which was used directly within the model calibration. In this section we make additional model-observation comparisons in order to validate the calibrated models resulting from each parameter estimation experiment. 


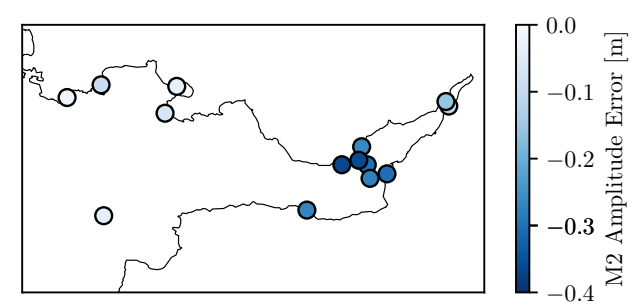

Fig. 9: Map of M2 amplitude model errors, using the 'standard' parameters. The errors increase in magnitude further into the channel.

\subsection{Validation using additional harmonic constituents}

The parameter estimation algorithm used only the M2 and S2 amplitude and phase data at the locations indicated by red circles in Fig. 1. As described in section 2.2, the N2 and M4 constituents have amplitudes in the $10 \mathrm{~s}$ of $\mathrm{cm}$ within the model domain. These constituents are included in the model boundary condition, and can be resolved by harmonic analysis based on the one-month model runs. We can therefore make additional comparisons between the modelled and observed amplitudes and phases for these two constituents. These RMSEs are summarised in Table 4.

The 'standard' sediment-based friction field produces the smallest N2 amplitude RMSE, in contrast with its poor performance on all other error metrics. The benchmark run (with uniform $n=0.025 \mathrm{~s} \mathrm{~m}^{-1 / 3}$ ) produces the smallest $\mathrm{N} 2$ phase errors. Experiment B produces the smallest RMSEs for the M4 amplitude, while experiment C2 produces the smallest M4 phase RMSE. As was the case for the error metrics against the calibration data, experiments $\mathrm{C} 1$ and $\mathrm{C} 2$ produce similar RMSEs. Overall, the N2 and M4 validation metrics do not strongly favour a particular parameter estimation experiment, and the $\mathrm{N} 2$ amplitude in particular appears difficult to model accurately.

Table 4: Root mean squared errors of the modelled N2 and M4 amplitudes and phases, for each Manning coefficient field, aggregated across the calibration tide gauges (red circles in Fig. 1). Figures in bold indicate the best performance.

\begin{tabular}{c|c|c|c|c}
\multirow{2}{*}{ Manning coefficient field } & \multicolumn{4}{|c}{ RMSE } \\
\cline { 2 - 5 } & $\mathrm{N} 2 \alpha[\mathrm{cm}]$ & $\mathrm{N} 2 \phi\left[^{\circ}\right]$ & $\mathrm{M} 4 \alpha[\mathrm{cm}]$ & $\mathrm{M} 4 \phi\left[^{\circ}\right]$ \\
\hline 'Standard' sediment-based parameters & $\mathbf{1 2 . 2}$ & 13.0 & 6.7 & 20.8 \\
Experiment A & 12.4 & 6.5 & 6.6 & 17.9 \\
Experiment B & 13.4 & 6.0 & $\mathbf{5 . 4}$ & 20.7 \\
Experiment C1 & 12.6 & 6.1 & 5.8 & 17.9 \\
Experiment C2 & 12.5 & 6.2 & 5.7 & $\mathbf{1 7 . 5}$ \\
\hline Uniform $n=0.025 \mathrm{~s} \mathrm{~m}^{-1 / 3}$ & 13.2 & $\mathbf{4 . 9}$ & 6.0 & 19.0
\end{tabular}


5.2 Validation using additional tide gauge locations

In this section we compare model outputs with data from the five BODC tide gauge locations (indicated by yellow circles in Fig. 1). Data at these locations were not used in the parameter estimation experiments.

The M2 and S2 amplitude and phase RMSEs aggregated across these five tide gauges are summarised in Table 5 for each BFC field. We find that experiment $\mathrm{C} 1$ produces the smallest values for all four RMSEs. Experiments A and C2 also perform well. Experiment B produces a relatively high M2 amplitude RMSE, but is still an improvement on the benchmark $n=$ $0.025 \mathrm{~s} \mathrm{~m}^{-1 / 3}$ run. Model performance for the N2 and M4 constituents at these validation tide gauges follows a similar pattern to the performance at the calibration gauges, and is therefore not shown.

Table 5: Root mean squared errors of the modelled M2 and S2 amplitudes and phases, for each Manning coefficient field, aggregated across the validation tide gauges (yellow circles in Fig. 1). Figures in bold indicate the best performance.

\begin{tabular}{c|c|c|c|c}
\multicolumn{2}{c}{} & \multicolumn{4}{|c}{ RMSE } \\
\cline { 2 - 5 } Manning coefficient field & $\mathrm{M} 2 \alpha[\mathrm{cm}]$ & $\mathrm{M} 2 \phi\left[^{\circ}\right]$ & $\mathrm{S} 2 \alpha[\mathrm{cm}]$ & $\mathrm{S} 2 \phi\left[^{\circ}\right]$ \\
\hline 'Standard' sediment-based parameters & 26.2 & 7.9 & 13.6 & 7.9 \\
Experiment A & 3.3 & 1.7 & 1.9 & 1.2 \\
Experiment B & 6.2 & 1.8 & 2.7 & 1.8 \\
Experiment C1 & $\mathbf{2 . 6}$ & $\mathbf{1 . 4}$ & $\mathbf{1 . 7}$ & $\mathbf{0 . 7}$ \\
Experiment C2 & 3.5 & 1.6 & 2.1 & $\mathbf{0 . 7}$ \\
\hline Uniform $n=0.025 \mathrm{~s} \mathrm{~m}^{-1 / 3}$ & 8.0 & 1.8 & 3.7 & 3.8
\end{tabular}

These results suggest that over-fitting has not been an issue in any of the parameter estimation experiments. The N2 and M4 error metrics do not strongly favour any particular BFC configuration, while the M2 and S2 error metrics at new locations show improvements which are consistent with the corresponding error metrics against the calibration data.

Due to the similarity in the results of experiments $\mathrm{C} 1$ and $\mathrm{C} 2$, throughout the remainder of this paper we limit our analysis to the 'standard' sediment-based parameters, and the results from parameter estimation experiments $\mathrm{A}, \mathrm{B}$ and $\mathrm{C} 1$.

\section{Implications for tidal range energy}

In this section, we consider the mean modelled tidal range energy, and its sensitivity to the bottom friction parameterisation. At a given location, the mean tidal range energy density (or potential energy density, PED) is computed as

$$
\mathrm{PED}=\frac{1}{M} \sum_{i=1}^{M} \frac{1}{2} \rho g\left(H W_{i}-L W_{i}\right)^{2},
$$

where the sum is over $M=28$ semidiurnal tidal periods spanning a single complete springneap cycle, $\rho$ is the density of water, and $H W_{i}$ and $L W_{i}$ are the high and low water surface elevations from each semidiurnal cycle $i$, respectively. The result has units of $\mathrm{J} \mathrm{m}^{-2}$ per tidal cycle. 
We compute the mean tidal range energy density at each of the tide gauge locations shown in Fig. 1, using both the model (with various friction parameters) and observations. This energy density is computed from surface elevation timeseries reconstructed from the M2 and S2 harmonic constituents, since these constituents dominate the tidal dynamics in the region and are well captured by the model. A comparison between these modelled and observed values is presented in Fig. 10. The 'standard' sediment parameters result in a severe underestimate of the tidal range energy density, while the other parameter sets all perform reasonably well. As shown in Table 6 , experiment $\mathrm{C} 1$ produces the smallest tidal range energy density RMSE; this is to be expected, since it also performs best in terms of M2 and S2 amplitude and phase RMSEs.

Fig. 11 shows the modelled mean tidal range energy density, computed over the entire Bristol Channel, using the BFC field from experiment C1. Fig. 12 shows the difference between the modelled mean tidal range energy density for each other BFC field, and the result from $\mathrm{BFC}$ field $\mathrm{C} 1$ (we use the model result from experiment $\mathrm{C} 1$ as a central value for these different plots since it has the lowest RMSE with respect to the available observations). The results are consistent with those of Fig. 10, and the spatial patterns can be explained by the BFC distributions shown in Fig. 7. Fig. 12(a) again demonstrates the under-estimation of the available tidal range energy when using the 'standard' sediment-based parameters. Fig. 12(b) shows that the uniform parameter tends to overestimate the tidal range energy density compared with parameters $\mathrm{C} 1$, particularly in the central part of the channel. This central region largely coincides with the presence of bedrock, i.e. where the BFC within experiment $\mathrm{A}$ is smaller than within $\mathrm{C} 1$, leading to the observed difference. This pattern is largely reversed in Fig. 12(c), corresponding to the difference between experiments B and $\mathrm{C} 1$; experiment $\mathrm{B}$ produces larger values for the $\mathrm{BFC}$ in the central rocky region than experiment $\mathrm{C} 1$, and therefore produces smaller modelled sea surface elevations. Towards the east end of the model domain (further upstream), the relative values of the BFCs are reversed, leading to a change in sign in the tidal range energy difference plots. Overall, these results reveal that the BFC has a somewhat localised effect on the modelled tidal range energy density, although the long tidal wavelength means that the differences in tidal range energy are much smoother than the differences between the BFC fields themselves (which are piecewise-constant and discontinuous).

Table 6: Root mean squared errors (RMSEs) of the modelled mean tidal range energy densities, compared with observations at the tide gauge locations.

\begin{tabular}{c|c} 
Manning coefficient field & $\mathrm{RMSE} / \mathrm{kJ} \mathrm{m}^{-2}$ \\
\hline 'Standard' parameters & 44.3 \\
Experiment A & 10.4 \\
Experiment B & 16.5 \\
Experiment C1 & 8.8
\end{tabular}

\section{Modelling tidal currents}

In this section we perform further model validation using available tidal current observations, and discuss the application of the calibrated model to tidal stream resource assessment. Freely available ADCP data is relatively scarce within the study region, but here we make comparisons with ADCP data collected at Minehead (shown as a purple diamond in Fig. 


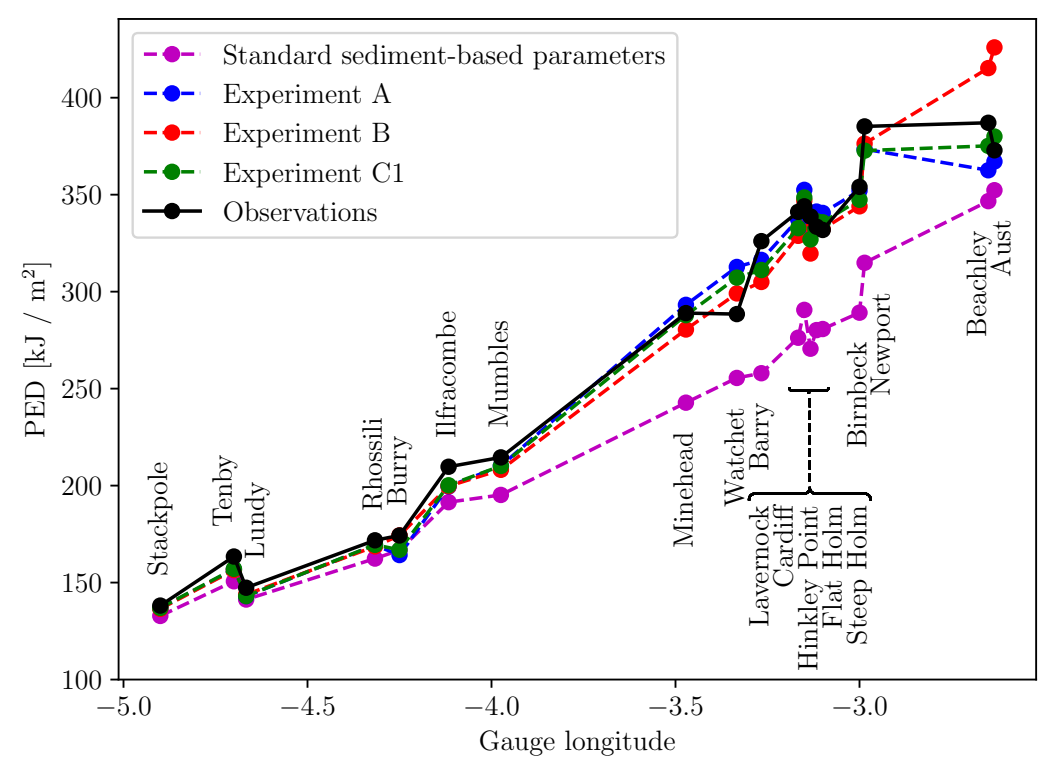

Fig. 10: Comparison of modelled and observed mean tidal range energy density over a spring-neap cycle. The names of each tide gauge location are indicated.

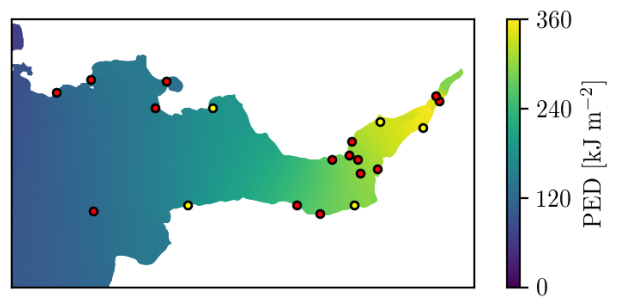

Fig. 11: Mean tidal range energy density per tidal cycle, computed over the entire Bristol Channel, using friction field $\mathrm{C} 1$ (spatially varying calibrated parameter). This field results in the smallest RMSEs vs observed tidal range energy density, and is therefore the best estimate of the tidal range energy resource across the Bristol Channel.

14), on $30^{\text {th }}$ July and $1^{\text {st }}$ August $2001[49,29]$. The ADCP measured velocity at 6 depths, and has been depth-averaged for numerical model comparisons.

Fig. 13 compares modelled and observed current speeds at the ADCP deployment location, for the four model BFC configurations. In all cases, the model overestimates the current speeds. One surprising result is that the 'standard' sediment parameters, which previous results suggest overestimate the bottom friction, produce the greatest modelled velocity magnitudes at the ADCP location. This can be explained by inspecting the friction coefficient distributions of Fig. 7. The sediment types within the region are shown in Fig. 14, with the ADCP location indicated. The large region of high friction coefficient in the centre of the 

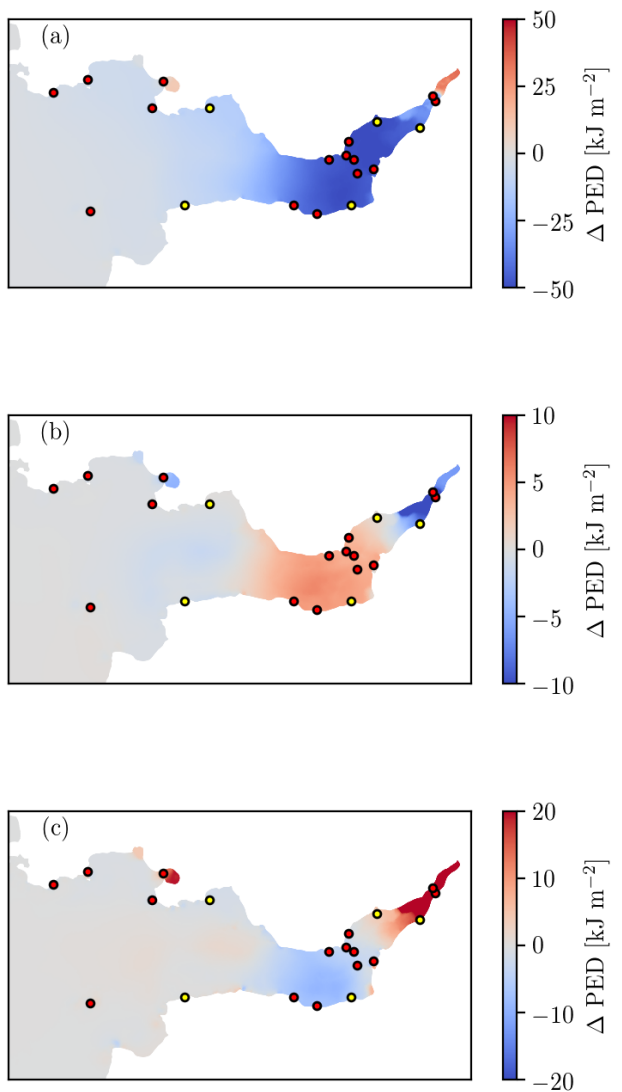

Fig. 12: Difference between modelled tidal range energy density (a) with 'standard' parameters and parameters from experiment $\mathrm{C} 1$; (b) with parameters from experiments $\mathrm{A}$ and $\mathrm{C} 1$; (c) with parameters from experiments $\mathrm{B}$ and $\mathrm{C} 1$. Note the different colorbar ranges in each figure. We again observe that the 'standard' sediment-based parameters underestimate the energy density compared with the calibrated parameters, by an increasing amount further into the Channel. In contrast, the uniform coefficient produces higher energy densities in the central bedrock region of the channel, since it does not impose higher friction here.

channel (corresponding to bedrock, sediment ID 1) acts to block the flow, driving higher currents along the southern edge of the model domain, where the sediments are finer and the BFC therefore smaller. This blockage effect depends on the relative friction coefficients between the bedrock region and the southern lower-friction area. Since the ADCP is situated within this lower friction region, the modelled velocities here are amplified by higher values for the bedrock friction coefficient. This explains why both the 'standard' sedimentbased friction parameters, and the result of experiment $\mathrm{B}$, produce the highest velocities at the ADCP location. For the parameters resulting from experiment $\mathrm{C} 1$, the $\mathrm{BFC}$ values are less extreme, and the blockage effect is therefore somewhat reduced. The parameters from experiment $\mathrm{A}$, corresponding to a uniform $\mathrm{BFC}$ within the Bristol Channel, result in the best 

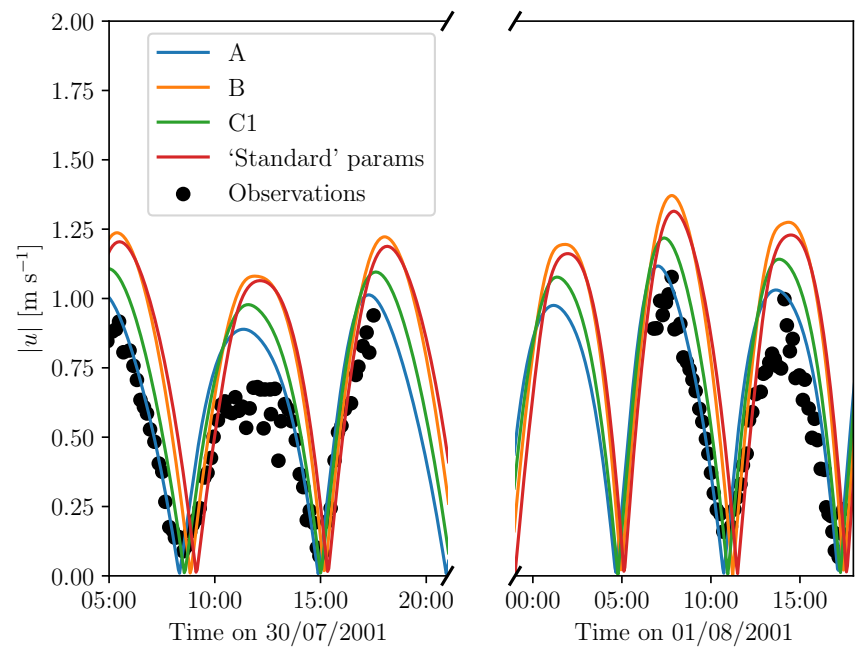

Fig. 13: Comparison between models with various friction parameters, and depth-averaged current speed data at Minehead ADCP, from [29].

performance at the ADCP location, because the uniform BFC removes the blockage effect altogether.

This is further demonstrated by Figs. 15 and 16. Fig. 15 shows the mean modelled kinetic power density (KPD) across the model domain, using the $\mathrm{C} 1$ parameters, and exhibits small-scale variability in the tidal stream resource, due mostly to bathymetric and coastline features. Similar to Fig. 12 for mean tidal range energy, Fig. 16 shows the differences between the modelled mean tidal stream power density for each BFC field, compared with the result from $\mathrm{BFC}$ field $\mathrm{C}$. There is high spatial correlation between these differences and the differences in the BFC fields (see Fig. 7), revealing a strongly localised effect of the BFC on the modelled tidal stream resource. In particular, Fig. 16b shows the difference in modelled mean tidal stream power density between parameters A (uniform BFC) and C1, and demonstrates the blockage effect described above, with the uniform BFC producing lower velocities in regions of finer sediment at the southern edge of the model domain.

Overall, the results of this section demonstrate the increased complexity of tidal currents compared with tidal elevations, with both the bathymetry and BFC having a strong localised effect on model velocities. We therefore conclude that calibration for tidal stream resource assessment requires further work. Tidal current observations spanning a broader spatial region are essential, and since currents are typically influenced by localised features that may well be underestimated in the interpolation of the bathymetry data to the unstructured mesh, the use of higher resolution in both the model mesh and the bathymetry may be required.

\section{Discussion}

This study has compared various uses of sedimentological data within BFC parameter estimation, using the Bristol Channel and Severn Estuary as a case study region. We have 


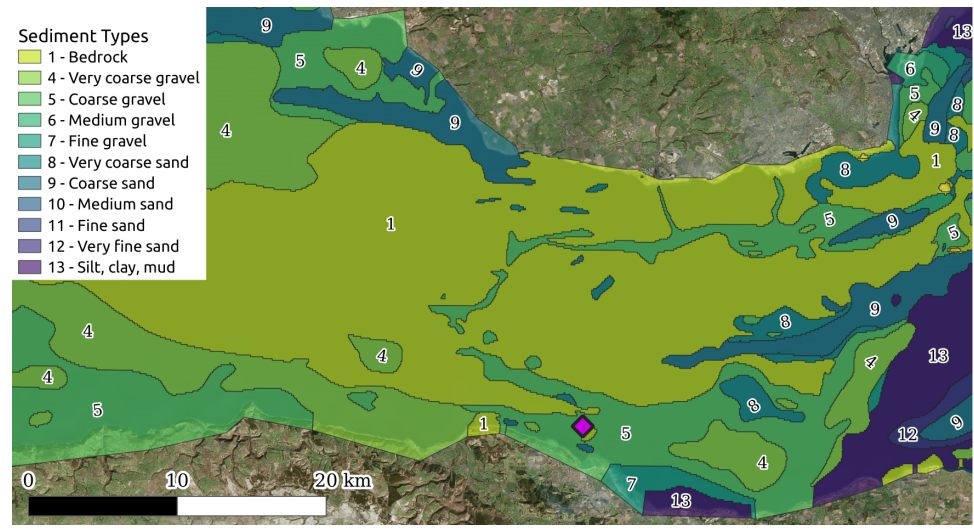

Fig. 14: Sediment zones, zoomed in to the central part of the channel. The purple diamond indicates the ADCP location, which lies within a region of relatively fine sediment.

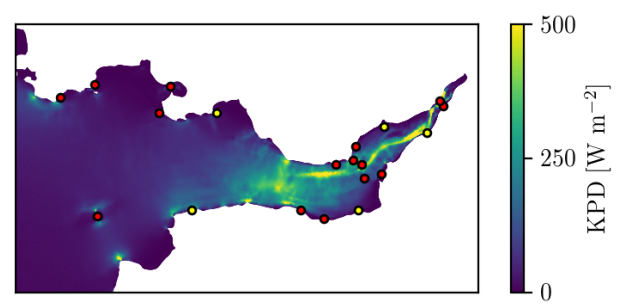

Fig. 15: Mean tidal stream power density, computed over the entire Bristol Channel, using friction parameters from experiment $\mathrm{C} 1$.

performed a number of parameter estimation experiments, utilising the sedimentological data in different ways. These calibration experiments can be considered to be zero-, oneand three-dimensional parameter estimation problems.

The use of 'standard' sediment-derived BFC parameters can be considered zero-dimensional, since this approach does not involve the use of any tide gauge data to infer any model parameters. Instead, theoretical values for the BFC were applied directly to the numerical model, based on the median grain size of the sediment found at each point within the model domain. This resulted in excessive friction parameters, leading to underestimation of tidal amplitudes. This is consistent with the presence of numerical diffusion within the model in addition to the bottom friction term within the governing equations; the optimal model BFCs are smaller than would be expected from the physics of the bottom friction effect [13].

Parameter estimation experiments A and B are both one-dimensional problems, but they take differing approaches. In experiment $\mathrm{A}$, a spatially uniform BFC was inferred, whereas in experiment $\mathrm{B}$ we took the sediment-derived $\mathrm{BFC}$ as a starting point, scaling the $\mathrm{BFC}$ by a uniform factor which was determined via the parameter estimation algorithm. Between these experiments, the uniform BFC (experiment A) produced better model performance, as measured against both the calibration and validation tide gauge data, than experiment $\mathrm{B}$. This implies that scaling by a constant factor is not sufficient to compensate for the shortcom- 

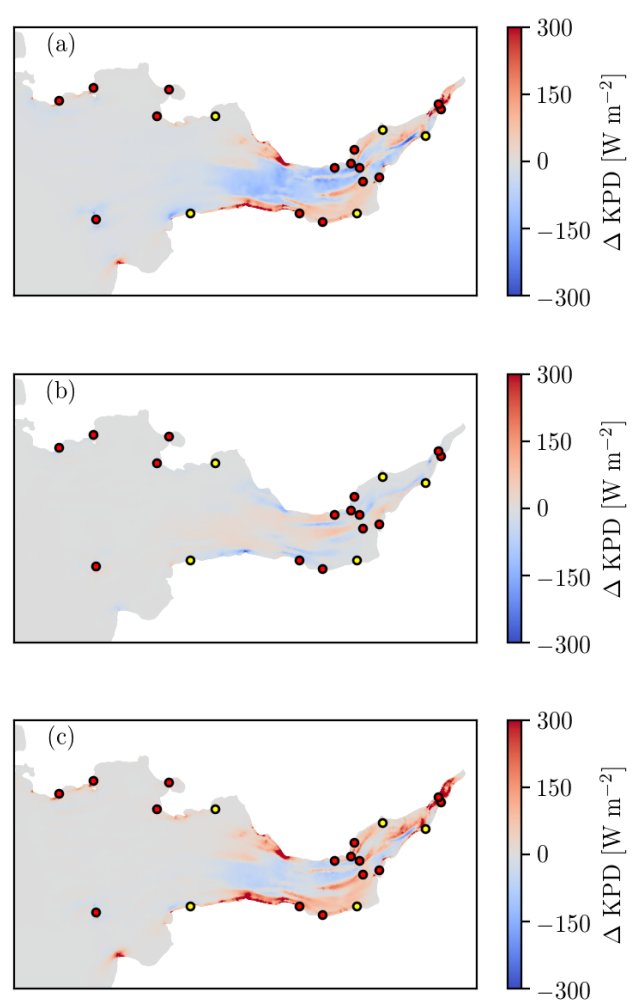

Fig. 16: (a) Difference in modelled mean tidal stream power density between 'standard' parameters and parameters C1. (b) Difference for parameters A and C1. (c): Difference for parameters B and C1. Similarly to the tidal range energy density, the tidal stream power density is mostly underestimated by the 'standard' sediment-based parameters compared with the calibrated parameters, with a particularly strong local effect in the region of bedrock in the channel centre. However, due to the blockage effect of increased friction in the centre of the channel, the kinetic energy increases at the southern edge, where the friction coefficient is smaller.

ings of the theoretical sediment-derived parameters, and therefore in modelling applications where there is insufficient data for estimating more than one parameter, or such calibration is considered unnecessary, the commonly-taken approach of a uniform BFC is most suitable. There may exist some function of the theoretical sediment-derived BFC (more complex than simple scaling as performed here) which can produce better model performance than a uniform BFC, but this would amount to the estimation of more than one parameter. The model performance with the optimal uniform BFC meets the recommended accuracy criteria of [59], and we therefore conclude that the estimation of a spatially uniform BFC is sufficient for many practical purposes. This may particularly be the case when using a calibration algorithm whose computational cost increases with the number of parameters to be estimated (such as the algorithm we use in this study), and given that reducing model errors under one metric may be liable to increase errors under another metric (such as is observed in this 
study, where the spatially varying BFCs, calibrated using tidal elevation data alone, perform worse in terms of tidal currents).

In experiments $\mathrm{C} 1$ and $\mathrm{C} 2$, the sedimentological data was used to divide the Channel into three subdomains, corresponding to groups of sediment types, and a Bayesian inference algorithm employed to estimate the optimal BFC corresponding to each sediment group. Experiments $\mathrm{C} 1$ and $\mathrm{C} 2$ differed in their choice of prior within the Bayesian inference; experiment $\mathrm{C} 1$ used a uniform prior, whereas experiment $\mathrm{C} 2$ used Gaussian priors based on the theoretical sediment-derived BFC values. Due to the increased dimension of the parameter space for experiments $\mathrm{C}$, their performance against both the calibration and validation tide gauge data was better than experiments $\mathrm{A}$ and $\mathrm{B}$. Overall, experiment $\mathrm{C} 1$ produced slightly better performance than experiment $\mathrm{C} 2$; this is further evidence that the theoretical $\mathrm{BFC}$ values derived from the sediment data are spurious in the context of numerical model BFCs, which may be due to the presence of other modelling errors. Nevertheless, the sediment data provides a physically motivated decomposition of the model domain for constraining the spatial variation of the friction parameter, for applications where there is sufficient observation data to calibrate the model with more than one degree of freedom.

This study did not investigate the use of BFC parameterisations with more than three degrees of freedom. Doing so could result in greater model performance, but could encounter overfitting issues, and is ultimately limited by the available observation data. Furthermore, since calibration implicitly compensates for a broad variety of modelling errors, calibration with respect to a greater number of degrees of freedom will arguably become increasingly disconnected from the underlying physics of the bottom friction effect, thus making the sedimentological data less useful in constraining the spatial variation of the BFC. The results of this study suggest that, for small-dimensional parameter estimation problems, the use of sediment data for subdividing the model domain constitutes a practical approach.

However, we acknowledge that even for the low-dimensional parameter spaces we considered here, the calibration problem will be affected by the presence of a variety of sources of error $[16,52]$. These sources include assumptions made within the governing equations (e.g. the choice between two- and three-dimensional models, barotropic vs baroclinic models, etc), discretisation errors, mesh resolution [20], unresolved bathymetry (e.g. sandbars [27]), other imperfect model inputs, and other unresolved or parameterised processes. However, reductions in each of these uncertainties typically incur additional computational cost, and/or require a greater volume of observation/survey data. The modelling approach and assumptions we have taken in this work are typical of many tidal range energy studies (including several utilising the same Thetis numerical model $[3,19,32,6])$, and we have sought to make the most of the available data. This study has also neglected temporal dependence of the BFC, e.g. within the spring-neap cycle, and has assumed calm conditions with no wind or atmospheric pressure forcing, or the propagation of storm surges from outside the model domain. On longer time scales, differences in the timing of observations may also be significant. For example, the sedimentological data used within this study was collected between 1977 and 1993, with the tide gauge observations also spanning multiple decades, whereas the bathymetry is likely to change on time scales of years to decades due to both anthropogenic and natural causes. Any calibrated BFC field is always specific to the model configuration with which it was derived, and model calibration should always be interpreted within the context of these other sources of model error. However, the use of spatiallydependent BFC is common within the literature (including within this model domain [33]). This study has attempted to make the most of limited data, demonstrating that sedimentological data can be an effective basis for constraining spatially varying BFCs. 
Within this work, we utilised M2 and S2 harmonic constituent data for model calibration. We acknowledge that the model-observation errors for these constituents are already small prior to calibration with a spatially varying BFC, given the broader context of the other modelling errors discussed above. However, this work has demonstrated that small changes in the BFC can correspond to changes in the tidal resonance, which is critical for the tidal dynamics and hence the tidal renewable energy resource. N2 and M4 data were withheld from the calibration, for the purposes of model validation. It is likely that incorporating all available data within the parameter estimation process would be beneficial, and may facilitate the estimation of a greater number of unknown parameters. We also note that the use of $\mathrm{N} 2$ data for validation was inconclusive in terms of differentiating model performance with each BFC field. Since the calibrated BFC fields will in part be compensating for imperfect model boundary conditions, the failure of M2- and S2-based calibration to improve the modelled N2 constituent may suggest the presence of errors in the boundary condition. It is certainly likely that calibration with respect to the boundary condition could produce additional improvements in model performance, but further investigation of this aspect is left to future work.

The results of section 6 reveal a somewhat localised effect of the BFC on the tidal range energy resource. This highlights the need for observations in regions of interest, although this is mitigated by the relatively smooth variation of tidal sea surface elevations. However, in an application to modelling tidal stream resource, the highly spatially variable nature of currents, which are affected by local coastline and bathymetry features, exacerbates this issue. Reliable tidal stream resource assessment therefore requires higher-density observations in regions of interest. The results of this study also suggest that the use of sediment types to parameterise the spatial variation of the friction parameter may not be appropriate when tidal currents are of interest. This is because the tidal currents are affected on small spatial scales by rapid changes in the BFC. We have also observed the BFC exerting a nonlocal effect on the tidal currents, where the use of high values for the BFC in the centre of the Channel drive higher currents along the southern edge of the Channel, where the BFC is lower. This blockage effect results in the counter-intuitive result that the 'standard' sediment-based BFC field, which results in underestimated sea surface heights, actually produces the highest current speeds at an ADCP situated near the southern edge of the Channel. Model calibration for tidal currents may require an alternative approach to BFC parameterisation which avoids sharp changes in the coefficient, e.g. via smoothing of the BFC field, or avoiding piecewise-constant BFC fields entirely. This aspect requires further work, and more extensive tidal current data.

\section{Conclusions}

This study has utilised sedimentological data within a numerical model of the Bristol Channel and Severn Estuary, in order to calibrate the model against available tide gauge data. The direct use of theoretical Manning coefficient values corresponding to the median grain size for each sediment type results in severe underestimates of the sea surface height, and consequently the tidal range energy resource. This can be improved by the reduction of these theoretical BFCs by scaling with a uniform factor, with the factor determined via a Bayesian inference algorithm. However, the resulting model performance can be further improved by the use of a well-selected spatially uniform BFC, confirming that when the data or computational resources permit the solution of only a one-dimensional parameter estimation problem, the spatially uniform BFC approach remains the best option. 
However, the results have demonstrated that the sedimentological data can be used to produce a piecewise-constant BFC according to three groups of sediment types. The solution of the resulting three-dimensional parameter estimation problem results in significant improvements in model performance over the uniform-BFC case, as measured against both the calibration and validation tide gauge data.

The application of the numerical model to tidal range resource assessment reveals a somewhat localised sensitivity to the BFC, highlighting the need for observation data in regions of interest. Due to the smaller-scale spatial variation in tidal currents, this issue is greater for tidal stream resource assessment, and we have also identified a non-local effect where excessive BFC values in the centre of the channel drive spuriously high currents 25 in other regions. This smaller-scale variation may also mean that the use of a piecewise26 constant BFC (such as the one used here based on dividing the domain by sediment types) 27 is incompatible with calibration for tidal currents, but further exploration of this issue is left to future work, and will require a larger volume of ADCP data.

Acknowledgements We acknowledge funding from the EPSRC, through the Centre for Doctoral Training in Fluid Dynamics across Scales (Grant EP/L016230/1), and grants EP/R029423/1 and EP/R511547/1. A. Angeloudis acknowledges the support of the NERC Industrial Innovation fellowship grant NE/R013209/2. We also acknowledge the Research Computing Service at Imperial College London for HPC resources and support. This study uses data from the National Tidal and Sea Level Facility, provided by the British Oceanographic Data Centre and funded by the Environment Agency.

\section{Conflict of interest}

The authors have no conflicts of interest to declare that are relevant to the content of this article.

\section{Data availability statement}

The datasets generated during and/or analysed during the current study are available from the corresponding author on reasonable request. 


\section{References}

1. Adcock, T.A.A., Draper, S., Nishino, T.: Tidal power generation-a review of hydrodynamic modelling. Proceedings of the Institution of Mechanical Engineers, Part A: Journal of Power and Energy 229(7), 755-771 (2015)

2. Angeloudis, A., Falconer, R.A.: Sensitivity of tidal lagoon and barrage hydrodynamic impacts and energy outputs to operational characteristics. Renewable Energy 114, 337-351 (2017)

3. Angeloudis, A., Kramer, S.C., Avdis, A., Piggott, M.D.: Optimising tidal range power plant operation. Applied energy 212, 680-690 (2018)

4. Arcement, G.J., Schneider, V.R.: Guide for Selecting Manning's Roughness Coefficients for Natural Channels and Flood Plains. Tech. rep. (1989). DOI ReportNo.FHWA-TS-84-204

5. Avdis, A., Candy, A.S., Hill, J., Kramer, S.C., Piggott, M.D.: Efficient unstructured mesh generation for marine renewable energy applications. Renewable Energy 116, 842-856 (2018). DOI https://doi.org/ 10.1016/j.renene.2017.09.058. URL http://www.sciencedirect.com/science/article/pii/ S0960148117309205

6. Baker, A.L., Craighead, R.M., Jarvis, E.J., Stenton, H.C., Angeloudis, A., Mackie, L., Avdis, A., Piggott, M.D., Hill, J.: Modelling the impact of tidal range energy on species communities. Ocean \& Coastal Management 193, 105221 (2020)

7. British Geological Survey: Seabed sediments $250 \mathrm{~K}$ https://www.bgs.ac.uk/datasets/marine-sediments250k/ (2021)

8. Chen, H., Cao, A., Zhang, J., Miao, C., Lv, X.: Estimation of spatially varying open boundary conditions for a numerical internal tidal model with adjoint method. Mathematics and Computers in Simulation 97, 14-38 (2014). DOI 10.1016/J.MATCOM.2013.08.005. URL https://www.sciencedirect.com/ science/article/pii/S0378475413002000

9. Davies, A., Robins, P.: Residual flow, bedforms and sediment transport in a tidal channel modelled with variable bed roughness. Geomorphology 295, 855-872 (2017)

10. Digimap: Marine Themes Digital Elevation Model 1 Arc Second [ASC geospatial data], Scale 1:50000, Tiles: 5050510045, 5050510050, 5051010030, 5051010035, 5051010040, 5051010045, 5051010050, 5051510025, 5051510030, 5051510035, 5051510040, 5051510045, 5051510050, Updated: 9 September 2016, OceanWise, Using: EDINA Marine Digimap Service, https://digimap.edina.ac.uk (2016)

11. Egbert, G.D., Erofeeva, S.Y.: Efficient inverse modeling of barotropic ocean tides. Journal of Atmospheric and Oceanic technology 19(2), 183-204 (2002)

12. Flather, R.A.: Existing operational oceanography. Coastal Engineering 41(1-3), 13-40 (2000)

13. Fringer, O.B., Dawson, C.N., He, R., Ralston, D.K., Zhang, Y.J.: The future of coastal and estuarine modeling: Findings from a workshop. Ocean Modelling 143, 101458 (2019)

14. Geuzaine, C., Remacle, J.F.: Gmsh: A 3-D finite element mesh generator with built-in pre- and postprocessing facilities. International Journal for Numerical Methods in Engineering 79(11), 1309-1331 (2009). DOI 10.1002/nme.2579

15. GPy: GPy: A Gaussian process framework in python. http://github.com/SheffieldML/GPy (since 2012)

16. Green, M.O., McCave, I.: Seabed drag coefficient under tidal currents in the eastern Irish Sea. Journal of Geophysical Research: Oceans 100(C8), 16057-16069 (1995)

17. Guillou, N., Thiébot, J.: The impact of seabed rock roughness on tidal stream power extraction. Energy 112, 762-773 (2016). DOI 10.1016/j.energy.2016.06.053

18. Hall, J.W., Manning, L.J., Hankin, R.K.: Bayesian calibration of a flood inundation model using spatial data. Water Resources Research 47(5), 1-14 (2011). DOI 10.1029/2009WR008541

19. Harcourt, F., Angeloudis, A., Piggott, M.D.: Utilising the flexible generation potential of tidal range power plants to optimise economic value. Applied Energy 237, 873-884 (2019)

20. Hasan, G.J., van Maren, D.S., Cheong, H.F.: Improving hydrodynamic modeling of an estuary in a mixed tidal regime by grid refining and aligning. Ocean Dynamics 62(3), 395-409 (2012)

21. Hastings, W.K.: Monte Carlo sampling methods using Markov chains and their applications. Biometrika 57(1), 97-109 (1970)

22. Heemink, A.W., Mouthaan, E.E.A., Roest, M.R.T., Vollebregt, E.A.H., Robaczewska, K.B., Verlaan, M.: Inverse 3D shallow water flow modelling of the continental shelf. Continental Shelf Research 22, 465-484 (2002)

23. Horsburgh, K.J., Wilson, C.: Tide-surge interaction and its role in the distribution of surge residuals in the North Sea. Journal of Geophysical Research 112(C8) (2007). DOI 10.1029/2006JC004033

24. Huybrechts, N., Smaoui, H., Orseau, S., Tassi, P., Klein, F.: Automatic Calibration of Bed Friction Coefficients to Reduce the Influence of Seasonal Variation: Case of the Gironde Estuary. Journal of Waterway, Port, Coastal, and Ocean Engineering 147(3), 05021004 (2021) 
25. Kärnä, T., de Brye, B., Gourgue, O., Lambrechts, J., Comblen, R., Legat, V., Deleersnijder, E.: A fully implicit wetting-drying method for DG-FEM shallow water models, with an application to the Scheldt Estuary. Computer Methods in Applied Mechanics and Engineering 200(5-8), 509-524 (2011). DOI 10.1016/j.cma.2010.07.001

26. Kärnä, T., Kramer, S.C., Mitchell, L., Ham, D.A., Piggott, M.D., Baptista, A.M.: Thetis coastal ocean model: Discontinuous Galerkin discretization for the three-dimensional hydrostatic equations. Geoscientific Model Development 11(11), 4359-4382 (2018). DOI 10.5194/gmd-11-4359-2018

27. Leuven, J., Kleinhans, M., Weisscher, S., Van der Vegt, M.: Tidal sand bar dimensions and shapes in estuaries. Earth-science reviews 161, 204-223 (2016)

28. Li, X., Plater, A., Leonardi, N.: Modelling the transport and export of sediments in macrotidal estuaries with eroding salt marsh. Estuaries and Coasts 41(6), 1551-1564 (2018)

29. Liang, D., Xia, J., Falconer, R.A., Zhang, J.: Study on tidal resonance in Severn Estuary and Bristol Channel. Coastal Engineering Journal 56(01), 1450002 (2014)

30. Lu, X., Zhang, J.: Numerical study on spatially varying bottom friction coefficient of a $2 \mathrm{D}$ tidal model with adjoint method. Continental Shelf Research 26(16), 1905-1923 (2006). DOI 10.1016/J.CSR.2006. 06.007. URL https://www.sciencedirect.com/science/article/pii/S027843430600210X

31. Lyddon, C., Brown, J.M., Leonardi, N., Plater, A.J.: Uncertainty in estuarine extreme water level predictions due to surge-tide interaction. PloS ONE 13(10), e0206200 (2018)

32. Mackie, L., Coles, D., Piggott, M., Angeloudis, A.: The potential for tidal range energy systems to provide continuous power: a uk case study. Journal of Marine Science and Engineering 8(10), 780 (2020)

33. Mackie, L., Evans, P.S., Harrold, M.J., Tim, O., Piggott, M.D., Angeloudis, A.: Modelling an energetic tidal strait: investigating implications of common numerical configuration choices. Applied Ocean Research 108, 102494 (2021)

34. Marshall, K.N., Kaplan, I.C., Hodgson, E.E., Hermann, A., Busch, D.S., McElhany, P., Essington, T.E., Harvey, C.J., Fulton, E.A.: Risks of ocean acidification in the California Current food web and fisheries: ecosystem model projections. Global Change Biology 23(4), 1525-1539 (2017)

35. Maßmann, S.: Tides on unstructured meshes. Ph.D. thesis, Universitat Bremen (2010)

36. Mayo, T., Butler, T., Dawson, C., Hoteit, I.: Data assimilation within the Advanced Circulation (ADCIRC) modeling framework for the estimation of Manning's friction coefficient. Ocean Modelling 76, 43-58 (2014). DOI 10.1016/j.ocemod.2014.01.001

37. Neill, S.P., Angeloudis, A., Robins, P.E., Walkington, I., Ward, S.L., Masters, I., Lewis, M.J., Piano, M., Avdis, A., Piggott, M.D., et al.: Tidal range energy resource and optimization-Past perspectives and future challenges. Renewable Energy 127, 763-778 (2018)

38. NERC: Surface Water and Ocean Topography (SWOT) satellite calibration and validation (cal/val). https://nerc.ukri.org/research/funded/programmes/ surface-water-and-ocean-topography-swot/, accessed 17/06/2021 (2021)

39. Periáñez, R., Casas-Ruíz, M., Bolívar, J.: Tidal circulation, sediment and pollutant transport in Cádiz Bay (SW Spain): a modelling study. Ocean Engineering 69, 60-69 (2013)

40. Proctor, R., Flather, R.: Storm surge prediction in the Bristol Channel-the floods of 13 December 1981. Continental Shelf Research 9(10), 889-918 (1989)

41. Quinn, N., Bates, P.D., Siddall, M.: The contribution to future flood risk in the Severn Estuary from extreme sea level rise due to ice sheet mass loss. Journal of Geophysical Research: Oceans 118(11), 5887-5898 (2013)

42. Rathgeber, F., Ham, D.A., Mitchell, L., Lange, M., Luporini, F., McRae, A.T., Bercea, G.T., Markall, G.R., Kelly, P.H.: Firedrake: Automating the finite element method by composing abstractions. ACM Transactions on Mathematical Software 43(3) (2016). DOI 10.1145/2998441

43. Siripatana, A., Mayo, T., Sraj, I., Knio, O., Dawson, C., Le Maitre, O., Hoteit, I.: Assessing an ensemble Kalman filter inference of Manning's $n$ coefficient of an idealized tidal inlet against a polynomial chaosbased MCMC. Ocean Dynamics 67(8), 1067-1094 (2017)

44. Sivia, D., Skilling, J.: Data analysis: a Bayesian tutorial. OUP Oxford (2006)

45. Smith, A., Porter, J.J., Upham, P.: "We cannot let this happen again": reversing UK flood policy in response to the Somerset Levels floods, 2014. Journal of Environmental Planning and Management 60(2), 351-369 (2017)

46. Soulsby, R.: Tidal current boundary layers, The Sea, Part 1. Edited by: B. Le Méhauté, University of Miami, USA, John Wiley \& Sons Inc., Printed in USA, ISBN: 0471633933 (1990)

47. Sraj, I., Iskandarani, M., Carlisle Thacker, W., Srinivasan, A., Knio, O.M.: Drag parameter estimation using gradients and hessian from a polynomial chaos model surrogate. Monthly Weather Review 142(2), 933-941 (2014). DOI 10.1175/MWR-D-13-00087.1

48. Sraj, I., Mandli, K.T., Knio, O.M., Dawson, C.N., Hoteit, I.: Uncertainty quantification and inference of Manning's friction coefficients using DART buoy data during the Tōhoku tsunami. Ocean Modelling 83, 82-97 (2014). DOI 10.1016/j.ocemod.2014.09.001 
49. Stapleton, C., Wyer, M., Kay, D., Bradford, M., Humphrey, N., Wilkinson, J., Lin, B., Yang, L., Falconer, R.A., Watkins, J., et al.: Fate and transport of particles in estuaries: Volume iv: Numerical modelling for bathing water enterococci estimation in the severn estuary (2007)

50. Vazquez, A., Iglesias, G.: LCOE (levelised cost of energy) mapping: A new geospatial tool for tidal stream energy. Energy 91, 192-201 (2015)

51. Vouriot, C.V., Angeloudis, A., Kramer, S.C., Piggott, M.D.: Fate of large-scale vortices in idealized tidal lagoons. Environmental Fluid Mechanics 19(2), 329-348 (2019). DOI 10.1007/s10652-018-9626-4. URL https ://doi.org/10.1007/s10652-018-9626-4

52. Waldman, S., Baston, S., Nemalidinne, R., Chatzirodou, A., Venugopal, V., Side, J.: Implementation of tidal turbines in MIKE 3 and Delft3D models of Pentland Firth \& Orkney Waters. Ocean \& Coastal Management 147, 21-36 (2017)

53. Wang, T., Yang, Z.: A Tidal Hydrodynamic Model for Cook Inlet, Alaska, to Support Tidal Energy Resource Characterization. Journal of Marine Science and Engineering 8(4), 254 (2020)

54. Warder, S.C., Horsburgh, K.J., Piggott, M.D.: Adjoint-based sensitivity analysis for a numerical storm surge model. Ocean Modelling 160, 101766 (2021)

55. Warder, S.C., Kramer, S.C., Piggott, M.D.: Non-deterministic effects in modelling the tidal currents in a high-energy coastal site. Submitted to Continental Shelf Research (2021). DOI 10.31223/X55G7F

56. Wessel, P., Smith, W.H.F.: A global, self-consistent, hierarchical, high-resolution shoreline database. Journal of Geophysical Research: Solid Earth 101(B4), 8741-8743 (1996). DOI 10.1029/96JB00104

57. Whomersley, P., Van der Molen, J., Holt, D., Trundle, C., Clark, S., Fletcher, D.: Modeling the dispersal of spiny lobster (Palinurus elephas) larvae: Implications for future fisheries management and conservation measures. Frontiers in Marine Science 5, 58 (2018)

58. Williams, J.A., Horsburgh, K.J.: Evaluation and comparison of the operational Bristol Channel model storm surge suite. Tech. rep., National Oceanography Centre (2013)

59. Williams, J.J., Esteves, L.S.: Guidance on setup, calibration, and validation of hydrodynamic, wave, and sediment models for shelf seas and estuaries. Advances in Civil Engineering 2017 (2017). DOI 10.1155/2017/5251902. Article ID 5251902

60. Zhang, J., Lu, X., Wang, P., Wang, Y.P.: Study on linear and nonlinear bottom friction parameterizations for regional tidal models using data assimilation. Continental Shelf Research 31(6), 555-573 (2011). DOI 10.1016/J.CSR.2010.12.011. URL https://www.sciencedirect.com/science/article/ pii/S0278434310003857 